\title{
A robust asset-liability management framework for investment products with guarantees
}

\author{
Nalan Gülpınar ${ }^{1}$. Dessislava Pachamanova ${ }^{2}$. \\ Ethem Çanakoğlu ${ }^{3}$
}

Received: 6 September 2014 / Accepted: 12 February 2016 / Published online: 28 March 2016 (C) The Author(s) 2016. This article is published with open access at Springerlink.com

\begin{abstract}
This paper suggests a robust asset-liability management framework for investment products with guarantees, such as guaranteed investment contracts and equity-linked notes. Stochastic programming and robust optimization approaches are introduced to deal with data uncertainty in asset returns and interest rates. The statistical properties of the probability distributions of uncertain parameters are incorporated in the model through appropriately selected symmetric and asymmetric uncertainty sets. Practical data-driven approaches for implementation of the robust models are also discussed. Numerical results using generated and real market data are presented to illustrate the performance of the robust asset-liability management strategies. The robust investment strategies show better performance in unfavorable market regimes than traditional stochastic programming approaches. The effectiveness of robust investment strategies can be improved by calibrating carefully the shape and the size of the uncertainty sets for asset returns.
\end{abstract}

Keywords Uncertainty modeling - Investment contracts with guarantees · Asset-liability management · Robust optimization · Stochastic programming

\footnotetext{
Nalan Gülpınar

Nalan.Gulpinar@wbs.ac.uk

Dessislava Pachamanova

dpachamanova@babson.edu

Ethem Çanakoğlu

ethem.canakoglu@bahcesehir.edu.tr

1 Warwick Business School, The University of Warwick, Coventry CV4 7AL, UK

2 Mathematics and Sciences Division, Babson College, Wellesley, MA 02457, USA

3 Industrial Engineering Department, Bahcesehir University, Istanbul, Turkey
} 


\section{Introduction}

Investment products with guarantees offer policyholders a guaranteed stream of payments and a portion of the potential gains on an underlying asset over a fixed period of time. Examples of such products include Guaranteed Investment Contracts (GICs), issued by insurance companies, and Equity-Linked Notes (ELNs), issued by investment banks. Investment products with guarantees provide a smoothing of portfolio returns to the policy (note) holders, so that the latter do not experience the full volatility of the underlying portfolio (Consiglio et al. 2006).

The basic structure of a typical GIC is as follows. The investor pays the "principal" upfront, and then receives a guaranteed rate of return over the life of the contract (Stiefel 1984). The last payment includes the value of the principal. Guaranteed investment contracts are popular investment vehicles-AIG notoriously used US $\$ 9$ billion of the government bailout after the crisis in the late 2000s to pay out on guaranteed investment contracts it had sold to investors (Walsh 2008). ELNs have similar terms to GICs. However, the payment stream is linked to the value of an equity security such as an equity index or a portfolio of assets. A portion of the returns generated by the equity index or the portfolio of assets over a specified period is paid to the policyholder (Ramaswami et al. 2001; Miltersen and Persson 2003; Toy and Ryan 2000). The principal is typically guaranteed, and hence the investor obtains fixed-income-like principal protection of his investment with an equity market upside exposure (Hardy 2003). At maturity, the guaranteed return and added bonuses along with the original capital invested are returned to the noteholder.

Firms issuing investment products with guarantees face an asset-liability management (ALM) problem. On the one hand, they need to invest the available capital (assets) collected from the principal payments profitably. On the other hand, they need to manage their obligations (liabilities) to policy holders. Insurance companies issuing GICs typically take a different approach from banks issuing ELNs. The former pool the premiums from the policy holders and invest them in a portfolio with a substantial equity component (see Consiglio et al. 2006) or a fixed income component (see Chapter 15 in Pachamanova and Fabozzi 2016). The latter typically hedge their exposure by purchasing exotic options or combinations of financial derivatives.

In this paper, we focus on the particular problem faced by an issuer of an investment product with guarantees that would like to determine the optimal structure of an underlying equity portfolio so as to maximize net portfolio return while meeting liabilities. This problem has not been addressed much in the literature. At the same time, a substantial amount of research has been directed at solving the pricing problem for investment contracts with guarantees-namely, determining the best guaranteed rate of return and optimal values for other contract features. In solving the pricing problem, the underlying portfolio is assumed to be given exogenously rather than structured optimally. For example, going as far back as the 1970s, Brennan and Schwartz (1976) determine the equilibrium pricing of equity-linked life insurance policies with an asset value guarantee. Brennan and Schwartz (1979) discuss investment strategies for equity-linked life insurance policies with an asset value guarantee. Mallier and Alobaidi (2002) develop a Vasicek model to price equitylinked notes where the holder receives both interest payments and payments linked to 
the performance of an equity index. Bacinello (2003) studies the problem of pricing a participating policy sold in the Italian market using guaranteed investment contracts. Nietert (2003) investigates option based portfolio insurance and model uncertainty. As shown by Consiglio et al. (2001), however, firms can substantially increase their profits and offer higher guarantees by investing a higher proportion of their assets in an optimally structured equity portfolio. Consiglio et al. (2006) apply stochastic programming to find the optimal structure of the portfolio underlying an insurance company's fund. Consiglio et al. (2008) discuss various issues with asset and liability modeling for participating policies with guarantees. Valle et al. (2014) develop a mixed integer optimization model for a portfolio of assets that is designed to deliver a constant return per time period irrespective of how the underlying market performs.

We propose a robust optimization approach to structuring the optimal portfolio for investment products with guarantees. The approach addresses two issues with previously suggested computational approaches to managing the underlying portfolio for GICs and ELNs: tractability and representation of the underlying uncertainties. While we are concerned with optimal allocation, the proposed approach can potentially have applications in the pricing of such contracts as well. As we mentioned, the current literature on pricing assumes that the structure of the portfolio is given exogenously. Because this approach is computationally efficient and tractable, acceptable values for the parameters of the contract can be derived by solving the optimal structuring problem multiple times to determine the parameters that will result in the highest profit for the issuer of the contract.

Robust optimization was first introduced by Ben-Tal and Nemirovski (1998) and El Ghaoui and Lebret (1997). Since then, it has been applied for solving various practical problems in different areas. The robust optimization approach assumes that the uncertain parameters in an optimization problem belong to uncertainty sets that can be constructed from the probability distributions of uncertain factors. A robust counterpart of the original problem requires that the optimal solution to the optimization problem remain feasible for all realizations of the stochastic data within the pre-specified uncertainty sets, including the worst-case values if they can be found. Depending on the specification of the uncertainty sets, the robust counterparts of the original optimization problems can be formulated as tractable optimization problems with no random parameters. For further information on robust optimization and recent developments, the reader is referred to Ben-Tal et al. (2009).

Robust optimization applications in finance have been primarily in asset management (for a comprehensive overview, see Fabozzi et al. 2007). The robust mean-variance portfolio selection framework has been widely studied; see, for instance, Goldfarb and Iyengar (2003), Gulpinar and Rustem (2007), Oguzsoy and Guven (2007), and Soyster and Murphy (2013). Robust investment strategies in a multiperiod setting are studied in Ben-Tal et al. (2000) and Bertsimas and Pachamanova (2008). Pinar (2007) studies a robust scenario-optimization-based downside risk measure for multi-period portfolio selection. Pae and Sabbaghi (2014) consider log-robust portfolios after transaction costs. Gulpinar and Pachamanova (2013) develop a robust ALM model for a pension fund with time-varying asset returns using ellipsoidal uncertainty sets mapped from a time series model for asset returns. The ALM model for a 
typical pension fund involves contributions from wages over the life of the contract, and the liabilities are paid from the fund.

This paper makes three main contributions to the literature. First, we show how the multi-period allocation problem for equity portfolios underlying investment products with guarantees can be cast in a robust multi-period optimization framework. Second, we are able to incorporate asymmetries in the distribution of asset returns in this framework. The latter is important for practical implementation because there is substantial empirical evidence that asset returns are not symmetrically distributed (see, for example, the discussion in Natarajan et al. 2008). Third, we suggest a scenario-based data-driven approach for estimating the input parameters in the robust formulations. We design numerical experiments to illustrate the performance of the robust ALM models under different assumptions on the behavior of the underlying uncertainties. We also compare the performance of robust ALM investment strategies with the performance of expected value optimization using generated and real market data. By taking a worst-case view, the robust optimization approach to asset-liability management of investment products with guarantees allows for incorporating new ways to analyze the performance of investment policies that is even more important in the aftermath of the financial crisis of 2007-2008. At the same time, computational tractability and the ability to incorporate the asymmetry in asset returns in the models make robust optimization formulations to multi-period asset management of the underlying portfolios for investment products with guarantees an attractive and useful tool in the investment manager's toolbox.

The paper is organized as follows. In Sect. 2, we introduce the ALM problem for investment products with guarantees. Section 3 presents a scenario-based stochastic programming model. Robust formulations of ALM models using symmetric and asymmetric uncertainty sets are developed in Sect. 4. Practical suggestions on implementation and input estimation from data are provided in Sect. 5. Results from computational experiments are presented in Sect. 6. Section 7 summarizes our findings.

Notation: We use tilde ( $\tilde{*})$ to denote randomness; e.g., $\tilde{z}$ denotes random variable $z$. Boldface is used to denote vectors; boldface and capital letters are used to denote matrices. For example, $\boldsymbol{a}$ is a vector and $\boldsymbol{A}$ is a matrix. A description of the notation used in the paper is provided in Table 1.

\section{Problem statement}

We are concerned with the following ALM problem for a company that issues investment products with guarantees. The company has certain obligations to policyholders and the liabilities of the company are determined by the underlying investment products. The holder of a policy gets a fixed guaranteed return and, in addition, a variable reversionary bonus. A bonus allows the policyholder to participate in the investment returns of the company. The issuer of the product needs to ensure that the asset allocation is capable of generating a surplus wealth at the end of the planning horizon to cover the liabilities.

We assume that the investment portfolio is constructed from $M$ risky assets over a planning horizon $T$. Securities are denoted by $m=1,2, \ldots, M$, and $m=0$ identifies 
Table 1 Description of notation

\begin{tabular}{ll}
\hline Parameters & Target funding (asset/liability) ratio \\
\hline$\psi$ & Transaction costs for buying and selling, respectively \\
$c_{\mathrm{b}}, c_{\mathrm{S}}$ & Amount (liabilities) paid out at time $t$ \\
$l_{t}$ & Guaranteed rate of return per period \\
$\bar{g}$ & Coupon payment at $t$ \\
$C_{t}$ & Capital (principal) paid \\
$P$ & \\
\hline Decision variables & Holding in asset $m$ at time $t$ \\
\hline$h_{t}^{m}$ & Amount sold of asset $m$ at time $t$ \\
$s_{t}^{m}$ & Amount bought of asset $m$ at time $t$ \\
$b_{t}^{m}$ & \\
\hline Random variables & Return on asset $m$ between time $t-1$ and $t$ \\
\hline$\tilde{r}_{t}^{m}$ & Present value of the total amount of future outstanding liabilities at time $t$ \\
$\tilde{L}_{t}$ & Cumulative gross return on asset $m$ at $t$ \\
$\tilde{R}_{t}^{m}$ & \\
\hline &
\end{tabular}

the risk-free asset. After an initial investment at $t=0$, the portfolio may be restructured at discrete times $t=1, \ldots, T-1$ and redeemed at the end of the investment horizon (at $t=T$ ). Let $h_{t}^{m}, s_{t}^{m}$ and $b_{t}^{m}$ denote decision variables representing the amount of asset $m$ to be held, sold and bought at time $t$, respectively.

The ALM formulation contains two sets of uncertain parameters: the asset returns $\tilde{\boldsymbol{r}}_{t}$ (including the return on the riskless asset $\tilde{r}_{t}^{0}$ ) and the value of the future liabilities $\tilde{L}_{t}$ at each point in time $t$. The latter depends on the realized changes in interest rates between time 0 and time $t$.

Modeling liabilities: Let $\bar{g}$ denote the guaranteed minimum rate of return and $P$ be the principal. The bonus payment is determined according to a participation rate $\kappa$, which indicates the percentage of the portfolio return paid to policyholders. The participation rate is determined as a percentage of appreciation of the underlying equity that the policyholder receives. In addition to the guaranteed minimum rate of return, we consider a coupon payment $C_{t}$ at each time period $t$. The issuer aims to pay their liabilities at each time period in the future. The future liabilities at $t$ consist of the coupon payment as well as the fixed rate of the capital payment and are calculated as

$$
l_{t}=C_{t}+\bar{g} P, \quad t=1, \ldots, T-1 .
$$

The issuer's liability at the final time period under a no-bonus scheme is $l_{T}=C_{T}+\bar{g} P+P$.

The bonus is paid at maturity as a percentage of the excess returns over the promised rate (if the terminal wealth of the portfolio exceeds the guaranteed principal $P$ ). At maturity, the guaranteed return and added bonuses along with the original capital 
invested are returned to the holder of the product. Therefore, the liability of the company at maturity, $l_{T}$, is calculated as

$$
l_{T}=\max \left\{\kappa\left(\sum_{m=1}^{M} h_{T}^{m}+h_{T}^{0}-P\right), \bar{g} P\right\}+P
$$

where $\kappa$ is a constant. When $\kappa=0$, as is the case with classical GICs, the liability at maturity is obtained as $l_{T}=P+\bar{g} P$. In any case, one can think of the true liability to the company at time $T$ as $l_{T}=P+\bar{g} P$. The added bonuses $\kappa\left(\sum_{m=1}^{M} h_{T}^{m}+h_{T}^{0}-P\right)$ are paid out to policy holders only if the portfolio performs well. The bonuses do not need to be taken into consideration for the purposes of determining a safety margin when planning on meeting future liabilities.

The liabilities $l_{t}$ to be paid out at each stage $t$ are therefore known at time 0 ; however, the total present value at time $t$ of all future liabilities between $t$ and $T$ is unknown because changes in the discount rates over time affect the present value of the cash flows. The present value of the total amount of future outstanding liabilities at time $t$ is

$$
\tilde{L}_{t}=\sum_{j=t+1}^{T} \frac{l_{j}}{\left(1+\tilde{r}_{t+1}^{0}\right) \times \cdots \times\left(1+\tilde{r}_{j}^{0}\right)}, \quad t=1, \ldots, T-1 .
$$

Asset-liability ratio: The asset-liability ratio, also called the funding ratio, is defined as the ratio of assets to liabilities. Firms typically have internal funding ratio constraints that inject a safety margin to enable the meeting of future liabilities. The funding ratio constraint can be formulated as

$$
\sum_{m=0}^{M} h_{t}^{m} \geq \psi \tilde{L}_{t}, \quad t=1, \ldots, T-1
$$

where $\psi$ denotes the target funding ratio, typically around 0.9 or 1 . Substituting the value of future liabilities at time $t$, the funding ratio constraint becomes

$$
\begin{gathered}
\sum_{m=0}^{M} h_{t}^{m} \geq \psi\left(\sum_{j=t+1}^{T} \frac{C_{j}+\bar{g} P}{\left(1+\tilde{r}_{t+1}^{0}\right) \times \cdots \times\left(1+\tilde{r}_{j}^{0}\right)}+\frac{P}{\left(1+\tilde{r}_{t+1}^{0}\right) \times \cdots \times\left(1+\tilde{r}_{T}^{0}\right)}\right), \\
t=1, \ldots, T-1 .
\end{gathered}
$$

Asset and cash holdings: The holdings in each asset $m$ at time $t$ are computed in terms of the holdings and gains from trading in the previous time period $t-1$ as well as the trading at the current time period $t$ as follows:

$$
h_{t}^{m}=\left(1+\tilde{r}_{t}^{m}\right) h_{t-1}^{m}-s_{t}^{m}+b_{t}^{m}, \quad t=1, \ldots, T, m=1, \ldots, M
$$

At time $t=0$, the initial holding of risky asset $m$ is $h_{0}^{m} \geq 0$, and $h_{0}^{0}=P$ denotes the cash holdings. The amount of cash at $t$ consists of value of investment at $t-1$ 
plus cash received from position changes and deposits (or bonus) payments minus the current liabilities paid out at time $t$,

$$
h_{t}^{0}=\left(1+\tilde{r}_{t}^{0}\right) h_{t-1}^{0}+\sum_{m=1}^{M}\left(1-c_{\mathrm{s}}\right) s_{t}^{m}-\sum_{m=1}^{M}\left(1+c_{\mathrm{b}}\right) b_{t}^{m}-l_{t}, \quad t=1, \ldots, T \text {. }
$$

We assume that there is no borrowing and short sales at any time period. The holdings of asset $m$ at time $t$ are thus restricted to be nonnegative:

$$
h_{t}^{m} \geq 0, \quad t=1, \ldots, T, \quad m=0, \ldots, M
$$

There is no transaction at the final time period $t=T\left(s_{T}^{m}=b_{T}^{m}=0\right)$ as well as at initial time period $t=0\left(s_{0}^{m}=b_{0}^{m}=0\right)$. For transactions at intermediate time periods $1 \leq t \leq T-1$, the decision variables corresponding to the amount of asset $m$ to be bought or sold cannot be negative:

$$
s_{t}^{m} \geq 0, \quad b_{t}^{m} \geq 0, \quad t=1, \ldots, T-1, \quad m=1, \ldots, M
$$

The portfolio profit in terms of possible bonus payment at the end of investment horizon can be calculated as the total wealth gained from each asset minus the liability including the bonus payment at the final time period $T$.

The stochastic ALM model for investment products with guarantees maximizes the expected net profit at the end of investment horizon subject to the funding ratio, balance and non-negativity constraints, and can be formulated as follows:

$$
\begin{array}{ll}
\max _{\mathbf{h}, \mathbf{b}, \mathbf{s}} & E\left[\sum_{m=0}^{\mathrm{stoc}} h_{T}^{m}-\max \left\{\kappa\left(\sum_{m=0}^{M} h_{T}^{m}-P\right), \bar{g} P\right\}-P\right] \\
\text { s.t. } & \sum_{m=1}^{M} h_{t}^{m}+h_{t}^{0} \geq \psi\left(\sum_{j=t+1}^{T} \frac{C_{j}+\bar{g} P}{\left(1+\tilde{r}_{t+1}^{0}\right) \times \cdots \times\left(1+\tilde{r}_{j}^{0}\right)}+\frac{P}{\left(1+\tilde{r}_{t+1}^{0}\right) \times \cdots \times\left(1+\tilde{r}_{T}^{0}\right)}\right), \\
& h_{t}^{m}=\left(1+\tilde{r}_{t}^{m}\right) h_{t-1}^{m}-s_{t}^{m}+b_{t}^{m}, \quad t=1, \ldots, T, m=1, \ldots, M \quad t=1, \ldots, T-1 \\
& h_{t}^{0}=\left(1+\tilde{r}_{t}^{0}\right) h_{t-1}^{0}+\sum_{m=1}^{M}\left(1-c_{\mathrm{S}}\right) s_{t}^{m}-\sum_{m=1}^{M}\left(1+c_{\mathrm{b}}\right) b_{t}^{m}-\left(C_{t}+\bar{g} P\right), \quad t=1, \ldots, T \\
& h_{t}^{m} \geq 0, \quad t=1, \ldots, T, m=0, \ldots, M \\
& s_{t}^{m} \geq 0, b_{t}^{m} \geq 0, \quad t=1, \ldots, T-1, m=1, \ldots, M .
\end{array}
$$

The general formulation $\mathcal{P}^{\text {stoc }}$ can be thought of as a formulation that represents the typical optimal portfolio structure problem for ELNs. When $\kappa=0$ and the coupon payments are fixed as $C_{t}=0$ for $t=1, \ldots, T$, one obtains the ALM formulation for a standard GIC without bonus provisions. 
Next, we present stochastic programming and robust optimization formulations of the ALM problem for investment products with guarantees. We contrast those formulations with traditional expected value optimization, and compare all three approaches in the computational experiments in Sect. 6.

\section{Scenario-based asset-liability management model}

Stochastic programming models describe underlying uncertainties in optimization problems in view of expected value decision criteria. It assumes that the uncertain parameters in the optimization problems follow a known distribution. There are different methods to deal with uncertain data such as scenario-based stochastic programming and chance-constrained optimization. A scenario-based stochastic programming approach takes into account a finite number of realizations of the random variables and specifies the optimal decisions in view of these scenarios (Dantzig and Infanger 1993). Chance-constrained stochastic programming involves probabilistic constraints to control risk in decision making under uncertainty.

There is an extensive literature on allocation strategies for ALM based on stochastic programming techniques that optimize investment strategies over a set of generated scenarios for future asset returns and liabilities (see, for example, Klaassen 1998; Ziemba and Mulvey 1998; Kouwenberg 2001; Gondzio and Kouwenberg 2001; Consigli and Dempster 1998; Boender et al. 2005; Escudero et al. 2009; Ferstl and Weissensteiner 2011). Gerstner et al. (2008) propose a simulation approach to the ALM problem of life insurance products in particular. As we mentioned earlier, Consiglio et al. (2006) develop a scenario-based model for insurance products with guarantees.

Let us consider a finite number of realizations, $\omega_{t}=1, \ldots, S_{t}$, of uncertain parameters $\tilde{r}_{t}^{m}$ for $m=0, \ldots, M$ at time $t=1, \ldots, T$. The probability $\Pi_{\omega_{T}}$ of a scenario $\omega_{T} \in S_{T}$ at time $T$ is called path probability and computed as multiplication of probabilities of scenarios arising on the path from $t=0$ to $t=T$. The scenarios do not anticipate the future. In other words, all possible scenarios $r_{\omega_{t^{\prime}}}^{m}$ for $t^{\prime}=1, \ldots, t-1$ are known by the investor at time $t$.

An expected value optimization ( $\mathcal{P}^{\text {average }}$ ) would inject average values $\hat{r}_{t}^{m}$ of the random variables at time $t$ into the ALM model. Then the underlying problem is solved as a deterministic problem.

More generally, a scenario-based ALM stochastic optimization problem optimizing the expected value of the objective function also becomes a deterministic model in view of the predefined scenarios for $r_{\omega_{t}}^{m}$ and can be stated as follows:

$\left(\mathcal{P}^{\text {scen }}\right)$ :

$$
\begin{aligned}
& \max _{\mathbf{h}, \mathbf{b}, \mathbf{s}} \sum_{\omega_{T} \in S_{T}} \Pi_{\omega_{T}}\left[\sum_{m=0}^{M} h_{\omega_{T}}^{m}-\max \left\{\kappa\left(\sum_{m=0}^{M} h_{\omega_{T}}^{m}-P\right), \bar{g} P\right\}-P\right] \\
& \text { s.t. } \\
& \qquad \sum_{m=0}^{M} h_{\omega_{t}}^{m} \geq \psi\left(\sum_{j=t+1}^{T} \frac{C_{j}+\bar{g} P}{\left(1+r_{\omega_{t+1}}^{0}\right) \times \cdots \times\left(1+r_{\omega_{j}}^{0}\right)}+\frac{P}{\left(1+r_{\omega_{t+1}}^{0}\right) \times \cdots \times\left(1+r_{\omega_{T}}^{0}\right)}\right), \\
& \quad \omega_{t} \in S_{t}, t=1, \ldots, T-1
\end{aligned}
$$




$$
\begin{aligned}
& h_{\omega_{t}}^{m}=\left(1+r_{\omega_{t}}^{m}\right) h_{p\left(\omega_{t}\right)}^{m}-s_{\omega_{t}}^{m}+b_{\omega_{t}}^{m}, \quad m=1, \ldots, M, \omega_{t} \in S_{t}, t=1, \ldots, T \\
& h_{\omega_{t}}^{0}=\left(1+\tilde{r}_{\omega_{t}}^{0}\right) h_{p\left(\omega_{t}\right)}^{0}+\sum_{m=1}^{M}\left(1-c_{\mathrm{s}}\right) s_{\omega_{t}}^{m}-\sum_{m=1}^{M}\left(1+c_{\mathrm{b}}\right) b_{\omega_{t}}^{m}-\left(C_{t}+\bar{g} P\right), \\
& \omega_{t} \in S_{t}, t=1, \ldots, T \\
& h_{\omega_{t}}^{m} \geq 0, \quad \omega_{t} \in S_{t}, t=1, \ldots, T, m=0, \ldots, M \\
& s_{\omega_{t}}^{m} \geq 0, b_{\omega_{t}}^{m} \geq 0, \quad \omega_{t} \in S_{t}, \quad t=1, \ldots, T-1, m=1, \ldots, M
\end{aligned}
$$

where $p\left(\omega_{t}\right) \in S_{t-1}$ denotes the parent node of scenario $\omega_{t} \in S_{t}$. Notice that in the asset-funding ratio constraints, the liabilities at each time period are discounted through a path between the parent node $p\left(\omega_{t}\right)$ and $\omega_{j}$ for $j=t+1, \ldots, T$ of the scenario tree.

The problem of finding optimal ALM policies using scenario-based optimization can be computationally challenging to implement in practice. While computational advances and smart implementation can make the problem manageable (for example, IBM's Algorithmics software splits ALM scenario calculations so that some parts of them can be pre-calculated and the calculations are done in the cloud), the performance of scenario-based ALM models heavily depends on the number of scenarios, and scenario generation inherently involves estimation errors (see, for instance Gulpinar et al. 2004).

It is worthwhile to mention that there are alternative stochastic optimization techniques based on dynamic programming algorithms that require specific modelling skills using states and actions that correspond to random paths and decisions in the multi-stage stochastic programming setting. However, these models also suffer from the curse of dimensionality in the state and action spaces. To deal with this, simulationbased dynamic programming approaches have been developed to solve the underlying problem approximately using forward dynamic programming algorithms. They have also been successfully applied to real life applications. The reader is referred to Powell (2011) for an overview and various applications of approximate dynamic programming.

\section{Robust ALM for investment products with guarantees}

Stochastic programming enables the calculation of optimal policies under complex conditions. However, as mentioned in the previous section, there are two main issues with its application. One is the curse of dimensionality, which affects the computational tractability of the optimization problem formulations. The other is the difficulty of knowing the exact distributions of the uncertainties in the optimization model. To address these issues, in this section we introduce a robust approach to ALM for investment products with guarantees and derive the robust counterparts of the ALM problem with symmetric (ellipsoidal) and asymmetric uncertainty sets. The latter were suggested by Chen et al. (2007); see also Natarajan et al. (2008). The results of computational experiments designed to evaluate the performance of the robust formulations derived here are presented in Sect. 6. 
The robust counterpart of the problem $\left(\mathcal{P}^{\text {stoc }}\right)$ is a formulation in which every constraint with uncertain coefficients is replaced with a constraint requiring that the inequality is satisfied for all values of the uncertain coefficients within pre-specified uncertainty sets. In particular, it is satisfied for the worst-case value of the expression in the constraint over the possible values for the uncertain coefficients. We will show how the robust counterpart is formulated in detail but first, we make a convenient change of variables.

We adopt a variable transformation suggested by Ben-Tal et al. (2000) and use the cumulative returns. Representing the decision variables in terms of cumulative returns reduces the number of constraints in which the uncertain returns appear in the ALM problem $\left(\mathcal{P}^{\text {stoc }}\right)$. For example, the uncertain returns currently appear in all balance constraints. Introducing cumulative returns, we have a particular uncertain parameter in only one as opposed to multiple constraints. This helps us not only to avoid crossconstraint correlations of uncertain parameters, which are more difficult to model, but also to reduce the conservativeness of the robust counterpart solution.

Let us define cumulative gross returns, $\tilde{R}_{t}^{m}$ for asset $m=1, \ldots, M$ at time $t=1, \ldots, T$ as

$$
R_{0}^{m}=1, \quad \tilde{R}^{m_{1}}=\left(1+\tilde{r}_{1}^{m}\right), \ldots, \tilde{R}_{t}^{m}=\left(1+\tilde{r}_{1}^{m}\right)\left(1+\tilde{r}_{2}^{m}\right) \cdots\left(1+\tilde{r}_{t}^{m}\right) .
$$

Introducing new decision variables for assets $m=1, \ldots, M$ and time periods $t=1, \ldots, T$,

$$
\xi_{t}^{m}=\frac{h_{t}^{m}}{\tilde{R}_{t}^{m}}, \quad \eta_{t}^{m}=\frac{s_{t}^{m}}{\tilde{R}_{t}^{m}}, \quad \zeta_{t}^{m}=\frac{b_{t}^{m}}{\tilde{R}_{t}^{m}},
$$

and a free variable $v$ for the objective function, we can rewrite the ALM problem $\left(\mathcal{P}^{\text {stoc }}\right)$ for investment products with guarantees in terms of cumulative returns as follows:

$$
\begin{aligned}
& \begin{array}{l}
\left(\mathcal{P}^{\mathrm{stoc}(R)}\right): \\
\max _{\xi, \eta, \zeta} \tilde{\boldsymbol{R}}_{T}^{\prime} \xi_{T}+\tilde{R}_{T}^{0} \xi_{T}^{0}-\max \left\{\kappa\left(\tilde{\boldsymbol{R}}_{T}^{\prime} \xi_{T}+\tilde{R}_{T}^{0} \xi_{T}^{0}-P\right), \bar{g} P\right\}-P \\
\text { s.t. } \\
\qquad \sum_{m=1}^{M} \xi_{t}^{m} \tilde{R}_{t}^{m}+\tilde{R}_{t}^{0} \xi_{t}^{0} \geq \psi\left(\sum_{j=t+1}^{T} \frac{\left(C_{j}+\bar{g} P\right) \tilde{R}_{t}^{0}}{\tilde{R}_{j}^{0}}+\frac{P \tilde{R}_{t}^{0}}{\tilde{R}_{T}^{0}}\right), \quad t=1, \ldots, T-1 \\
\xi_{t}^{m}=\xi_{t-1}^{m}-\eta_{t}^{m}+\zeta_{t}^{m}, \quad t=1, \ldots, T, m=1, \ldots, M \\
\xi_{t}^{0}=\xi_{t-1}^{0}+\sum_{m=1}^{M}\left(1-c_{\mathrm{s}}\right) \frac{\tilde{R}_{t}^{m}}{\tilde{R}_{t}^{0}} \eta_{t}^{m}-\sum_{m=1}^{M}\left(1+c_{\mathrm{b}}\right) \frac{\tilde{R}_{t}^{m}}{\tilde{R}_{t}^{0}} \zeta_{t}^{m}-\frac{C_{t}}{\tilde{R}_{t}^{0}}-\frac{\bar{g} P}{\tilde{R}_{t}^{0}}, \quad t=1, \ldots, T \\
\xi_{t}^{m} \geq 0, \quad t=1, \ldots, T, m=0, \ldots, M \\
\eta_{t}^{m} \geq 0, \zeta_{t}^{m} \geq 0, \quad t=1, \ldots, T-1, m=1, \ldots, M
\end{array}
\end{aligned}
$$


Notice that after the transformation of the decision variables, the uncertain (cumulative) returns appear only in the cash constraints, as opposed to all balance constraints. They also appear in the objective function and the funding ratio constraint, as they did before the transformation.

To formulate the robust counterpart of problem $\left(\mathcal{P}^{\text {stoc }(R)}\right)$, we first need to define appropriate uncertainty sets for the uncertain parameters in the problem, which are all terms involving the asset returns $\tilde{R}_{t}^{m}$ and the risk-free returns $\tilde{R}_{t}^{0}$. We then find the robust counterpart of the original optimization problem, which is an optimization problem in which all constraints with uncertain coefficients are required to be satisfied for any value of the uncertain coefficients in the specified uncertainty sets.

When solving optimization problems with uncertain parameters using the robust optimization approach, the size of the specified uncertainty set is often related to guarantees on the probability that the constraint with uncertain coefficients will not be violated (see, for example, Bertsimas et al. 2004). The shape of the uncertainty set defines a risk measure on the constraints with uncertain coefficients (Natarajan et al. 2009). In practice, the shape is selected to reflect the modeler's knowledge of the probability distributions of the uncertain parameters, keeping in mind that, ideally, the robust counterpart problem should be efficiently solvable if the uncertainties are assumed to belong to that uncertainty set. The ellipsoidal uncertainty set, for example, defines a standard-deviation-like risk measure on the constraint with uncertain parameters, and in the case of linear optimization, results in a robust counterpart to the original problem that is a second order cone problem - a tractable optimization problem.

Next, we derive the robust counterparts of the ALM model for investment products with guarantees using symmetric and asymmetric uncertainty sets. The symmetric and asymmetric shapes of the uncertainty sets should allow us to map the uncertainty sets better to uncertain parameters with symmetric and skewed distributions. In order to simplify the problem statement, we use the following notation for the vectors of random variables in the objective function, the balance constraints, and the funding ratio constraints, respectively:

$$
\begin{aligned}
\tilde{\boldsymbol{\alpha}}= & \left(\tilde{R}_{T}^{0}, \ldots, \tilde{R}_{T}^{M}\right) \in \Re^{M+1}, \\
\tilde{\boldsymbol{\rho}}_{t}= & \left(\left(1-c_{\mathrm{s}}\right) \frac{\tilde{R}_{t}^{1}}{\tilde{R}_{t}^{0}}, \ldots,\left(1-c_{\mathrm{s}}\right) \frac{\tilde{R}_{t}^{M}}{\tilde{R}_{t}^{0}},-\left(1+c_{\mathrm{b}}\right) \frac{\tilde{R}_{t}^{1}}{\tilde{R}_{t}^{0}}, \ldots,-\left(1+c_{\mathrm{b}}\right) \frac{\tilde{R}_{t}^{M}}{\tilde{R}_{t}^{0}},\right. \\
& \left.\quad-\left(C_{t}+\bar{g} P\right) \frac{1}{\tilde{R}_{t}^{0}}\right) \in \Re^{(2 M+1)}, \\
\tilde{\boldsymbol{\mu}}_{t}= & \left(\tilde{R}_{t}^{0}, \ldots, \tilde{R}_{t}^{M},-\psi\left(C_{t}+\bar{g} P\right) \frac{\tilde{R}_{t}^{0}}{\tilde{R}_{t+1}^{0}}, \ldots,-\psi\left(C_{t}+\bar{g} P\right) \frac{\tilde{R}_{t}^{0}}{\tilde{R}_{T}^{0}},\right. \\
& \left.\quad-\psi P \frac{\tilde{R}_{t}^{0}}{\tilde{R}_{T}^{0}}\right) \in \mathfrak{R}^{(M+T-t+2)} .
\end{aligned}
$$

Let vectors $\hat{\boldsymbol{\alpha}}, \hat{\boldsymbol{\rho}}_{t}$, and $\hat{\boldsymbol{\mu}}_{t}$ denote the expected values of the random vectors $\tilde{\boldsymbol{\alpha}}, \tilde{\boldsymbol{\rho}}_{t}$, and $\tilde{\boldsymbol{\mu}}_{t}$, respectively. For instance, $\hat{\boldsymbol{\alpha}}=\left(E\left[\tilde{R}_{T}^{0}\right], \ldots, E\left[\tilde{R}_{T}^{M}\right]\right)$. Similarly, we define vectors 
of decision variables $\epsilon=\left(\xi_{T}^{0}, \ldots, \xi_{T}^{M}\right)^{\prime}, \pi_{t}=\left(\eta_{t}^{1}, \ldots, \eta_{t}^{M}, \zeta_{t}^{1}, \ldots, \zeta_{t}^{M}, 1\right)^{\prime}$, and $\boldsymbol{\tau}_{t}=\left(\xi_{t}^{0}, \ldots, \xi_{t}^{M}, 1, \ldots, 1,1\right)^{\prime}$. Using the new notation, the robust counterpart of problem $\left(\mathcal{P}^{\text {stoc }(R)}\right)$ can be written in a compact form as follows:

$$
\begin{aligned}
& \left(\mathcal{P}^{\mathrm{rob}(R)}\right): \\
& \max _{\boldsymbol{\epsilon}, \boldsymbol{\pi}_{t}, \boldsymbol{\tau}_{t}, v, \phi} v \\
& \text { s.t. } \quad v \leq \min _{\tilde{\boldsymbol{\alpha}} \in \mathcal{U}^{o}}\{\tilde{\boldsymbol{\alpha}} \cdot \boldsymbol{\epsilon}\}-\phi-P \\
& \quad \phi \geq \min _{\tilde{\boldsymbol{\alpha}} \in \mathcal{U}^{o}}\{\kappa(\tilde{\boldsymbol{\alpha}} \cdot \boldsymbol{\epsilon}-P)\} \\
& \phi \geq \bar{g} P \\
& \xi_{t}^{m}=\xi_{t-1}^{m}-\eta_{t}^{m}+\zeta_{t}^{m}, \quad t=1, \ldots, T, m=1, \ldots, M \\
& \xi_{t}^{0} \leq \xi_{t-1}^{0}+\min _{\tilde{\boldsymbol{\rho}}_{t} \in \mathcal{U}_{t}^{h}}\left\{\tilde{\boldsymbol{\rho}}_{t} \cdot \boldsymbol{\pi}_{t}\right\}, \quad t=1, \ldots, T \\
& 0 \leq \min _{\tilde{\boldsymbol{\mu}}_{t} \in \mathcal{U}_{t}^{f}}\left\{\tilde{\boldsymbol{\mu}}_{t} \cdot \boldsymbol{\tau}_{t}\right\} \quad t=1, \ldots, T-1 \\
& \xi_{t}^{m} \geq 0, \quad t=1, \ldots, T, m=0, \ldots, M \\
& \eta_{t}^{m} \geq 0, \zeta_{t}^{m} \geq 0, \quad t=1, \ldots, T-1, m=1, \ldots, M
\end{aligned}
$$

where $\mathcal{U}^{o}, \mathcal{U}_{t}^{h}$ and $\mathcal{U}_{t}^{f}$ are the uncertainty sets associated with the uncertain parameters in the objective function, balance constraints and funding ratio constraints, respectively. The computational tractability of $\mathcal{P}^{\operatorname{rob}(R)}$ depends on the type of the uncertainty sets. Once the uncertainty sets are specified, the inner minimization problems are solved to derive the corresponding robust counterpart.

\subsection{Symmetric uncertainty sets}

Consider symmetric (ellipsoidal) uncertainty sets involving the uncertain future asset returns $\tilde{R}_{t}^{m}, m=1, \ldots, M$, and riskless returns $\tilde{R}_{t}^{0}$ at each point in time $t$, $t=1, \ldots, T$. The uncertainty sets are specified in terms of the means vectors $\hat{\boldsymbol{\alpha}}, \hat{\boldsymbol{\rho}}_{t}, \hat{\boldsymbol{\mu}}_{t}$ and the covariance matrices $\boldsymbol{\Xi}^{\alpha}, \boldsymbol{\Xi}_{t}^{\rho}$ and $\boldsymbol{\Xi}_{t}^{\mu}$ of the vectors of random variables $\tilde{\boldsymbol{\alpha}}, \tilde{\boldsymbol{\rho}}_{t}$, $\tilde{\boldsymbol{\mu}}_{t}$ in the sets of constraints with uncertain coefficients as follows:

$$
\begin{aligned}
\mathcal{S} \mathcal{U}^{o} & =\left\{\tilde{\boldsymbol{\alpha}} \mid\left\|\left(\boldsymbol{\Xi}^{\alpha}\right)^{-\frac{1}{2}}(\tilde{\boldsymbol{\alpha}}-\hat{\boldsymbol{\alpha}})\right\|_{2} \leq \theta^{o}\right\}, \\
\mathcal{S} \mathcal{U}_{t}^{h} & =\left\{\tilde{\boldsymbol{\rho}}_{t} \mid\left\|\left(\boldsymbol{\Xi}_{t}^{\rho}\right)^{-\frac{1}{2}}\left(\tilde{\boldsymbol{\rho}}_{t}-\hat{\boldsymbol{\rho}}_{t}\right)\right\|_{2} \leq \theta_{t}^{h}\right\}, \quad t=1, \ldots, T \\
\mathcal{S U}_{t}^{f} & =\left\{\tilde{\boldsymbol{\mu}}_{t} \mid\left\|\left(\mathbf{\Xi}_{t}^{\mu}\right)^{-\frac{1}{2}}\left(\tilde{\boldsymbol{\mu}}_{t}-\hat{\boldsymbol{\mu}}_{t}\right)\right\|_{2} \leq \theta_{t}^{f}\right\}, \quad t=1, \ldots, T-1,
\end{aligned}
$$

where $\theta^{o}, \theta_{t}^{h}$ and $\theta_{t}^{f}$ determine the size of the corresponding uncertainty sets, and are referred to as the "robustness budget" or the "price of robustness". The size of the uncertainty set corresponds to the amount of protection against uncertainty the decision maker desires, and can often be linked to guarantees on the probability that the 
constraint with uncertain coefficients will not be violated. There is a tradeoff between the size of the uncertainty set and optimality-the larger the degree of protection against uncertainty desired, the worse the optimal value of the objective function of the robust counterpart.

In order to find the robust counterpart of $\left(\mathcal{P}^{\operatorname{rob}(R)}\right)$ using uncertainty sets $\mathcal{S U}^{o}, \mathcal{S U}_{t}^{h}$, and $\mathcal{S U}_{t}^{f}$, we first separate the expressions in the constraints into expressions with uncertain coefficients and expressions with certain coefficients. We solve the inner minimization problems (taking place in the constraints) to find the worst-case values of the terms involving uncertain coefficients when these uncertain coefficients vary in the given uncertainty sets.

Let us illustrate how one would derive the robust counterpart of the first constraint. The constraint

$$
v-\tilde{\boldsymbol{\alpha}} \cdot \boldsymbol{\epsilon}+\phi+P \leq 0
$$

should be satisfied even if the vector of uncertain parameters takes their worst-case values within the uncertainty set. The worst-case value of uncertain expression is attained when $\tilde{\boldsymbol{\alpha}} \cdot \boldsymbol{\epsilon}$ is at its minimum value for any $\tilde{\boldsymbol{\alpha}}$ selected from the set $\mathcal{S U}^{o}$. Therefore, the robust formulation of the first constraint,

$$
v-\min _{\tilde{\boldsymbol{\alpha}} \in \mathcal{S U}^{o}}\{\tilde{\boldsymbol{\alpha}} \cdot \boldsymbol{\epsilon}\}+\phi+P \leq 0,
$$

is obtained by solving the inner minimization problem

$$
\begin{array}{ll}
\min _{\tilde{\boldsymbol{\alpha}}} & \tilde{\boldsymbol{\alpha}} \cdot \boldsymbol{\epsilon} \\
\text { s.t. } & \left\|\left(\boldsymbol{\Xi}^{\alpha}\right)^{-\frac{1}{2}}(\tilde{\boldsymbol{\alpha}}-\hat{\boldsymbol{\alpha}})\right\|_{2} \leq \theta^{o}
\end{array}
$$

and by reinjecting the optimal solution,

$$
\left(\tilde{\boldsymbol{\alpha}}^{*}\right)^{\prime} \cdot \boldsymbol{\epsilon}=\hat{\boldsymbol{\alpha}}^{\prime} \boldsymbol{\epsilon}-\theta^{o} \sqrt{\boldsymbol{\epsilon}^{\prime} \boldsymbol{\Xi}^{\alpha} \boldsymbol{\epsilon}},
$$

into the constraint. The robust counterparts of the remaining constraints in $\left(\mathcal{P}^{\operatorname{rob}(R)}\right)$ are derived in the same manner. They all include the expected values of the expressions with uncertain coefficients, as well as penalty-like terms that are related to the uncertain coefficients' standard deviations.

Based on the discussion so far, we can obtain the robust counterpart of $\left(\mathcal{P}^{\operatorname{rob}(R)}\right)$ under uncertainty sets $\mathcal{S U}^{o}, \mathcal{S U}_{t}^{h}$ and $\mathcal{S U}_{t}^{f}$ for the uncertain parameters $\tilde{\boldsymbol{\alpha}}, \tilde{\boldsymbol{\rho}}_{t}, \tilde{\boldsymbol{\mu}}_{t}$ as follows:

$$
\begin{aligned}
\underset{\boldsymbol{\epsilon}, \boldsymbol{\pi}_{t}, \boldsymbol{\tau}_{t}, v, \phi}{\left(\mathcal{P}^{s y m}\right):} & v \\
\text { s.t. } & v \leq \hat{\boldsymbol{\alpha}}^{\prime} \boldsymbol{\epsilon}-\theta^{o} \sqrt{\boldsymbol{\epsilon}^{\prime} \boldsymbol{\Xi}^{\alpha} \boldsymbol{\epsilon}}-\phi-P
\end{aligned}
$$




$$
\begin{aligned}
& \phi \geq \kappa\left(\hat{\boldsymbol{\alpha}}^{\prime} \boldsymbol{\epsilon}-\theta^{o} \sqrt{\boldsymbol{\epsilon}^{\prime} \boldsymbol{\Xi}^{\alpha} \boldsymbol{\epsilon}}-\phi-P\right) \\
& \phi \geq \bar{g} \cdot P \\
& \xi_{t}^{m}=\xi_{t-1}^{m}-\eta_{t}^{m}+\zeta_{t}^{m}, \quad t=1, \ldots, T, m=1, \ldots, M \\
& \xi_{t}^{0} \leq \xi_{t-1}^{0}+\hat{\boldsymbol{\rho}}_{t}^{\prime} \boldsymbol{\pi}_{t}-\theta_{t}^{h} \sqrt{\boldsymbol{\pi}_{t}^{\prime} \boldsymbol{\Xi}_{t}^{\rho} \boldsymbol{\pi}_{t}}, \quad t=1, \ldots, T \\
& 0 \leq \hat{\boldsymbol{\mu}}_{t}^{\prime} \boldsymbol{\tau}_{t}-\theta_{t}^{f} \sqrt{\boldsymbol{\tau}_{t}^{\prime} \boldsymbol{\Xi}_{t}^{\mu} \boldsymbol{\tau}_{t}}, \quad t=1, \ldots, T-1 \\
& \xi_{t}^{m} \geq 0, \quad t=1, \ldots, T, m=0, \ldots, M \\
& \eta_{t}^{m} \geq 0, \zeta_{t}^{m} \geq 0, \quad t=1, \ldots, T-1, m=1, \ldots, M
\end{aligned}
$$

Note that for the ellipsoidal uncertainty sets that are described in terms of the means and the covariance matrices of the uncertain coefficients, the robust counterparts of the constraints include the expected values of the expressions with uncertain coefficients, as well as penalty-like terms that are related to their standard deviations. Therefore, the robust counterpart to the original ALM problem $\left(\mathcal{P}^{\text {sym }}\right)$ becomes a second order cone program that is a tractable optimization problem.

\subsection{Asymmetric uncertainty sets}

Symmetric uncertainty sets can represent uncertainties well when these uncertainties follow symmetric probability distributions such as the normal distribution. As it has been shown empirically, however, both short- and long-horizon stock returns can be skewed and highly leptokurtic (see, for example, Duffee 2002). Chen et al. (2007) define measures of backward and forward deviation of probability distributions to allow for representing possible asymmetries in the uncertain cumulative returns better.

The forward and the backward deviation measures for a random variable $\tilde{z}$ are defined as $p(\tilde{z})=\inf \{\mathcal{P}(\tilde{z})\}$ and $q(\tilde{z})=\inf \{\mathcal{Q}(\tilde{z})\}$, where (see Chen et al. 2007):

$$
\mathcal{P}(\tilde{z})=\left\{\gamma: \gamma>0, \mathrm{E}\left(\exp \left(\frac{\phi}{\gamma} \tilde{z}\right)\right) \leq \exp \left(\frac{\phi^{2}}{2}\right) \forall \phi>0\right\},
$$

and

$$
\mathcal{Q}(\tilde{z})=\left\{\beta: \beta>0, \mathrm{E}\left(\exp \left(-\frac{\phi}{\beta} \tilde{z}\right)\right) \leq \exp \left(\frac{\phi^{2}}{2}\right) \forall \phi>0\right\} .
$$

It can be shown (Chen et al. 2007) that for a random variable $\tilde{z}$ with zero mean, $p(\tilde{z})$ and $q(\tilde{z})$ are always greater than or equal to the standard deviation of the distribution. In general, $p(\tilde{z})$ and $q(\tilde{z})$ are finite if the support $[-\underline{z}, \bar{z}]$ of the distribution for $\tilde{z}$ is finite. If the support is infinite, $p(\tilde{z})$ and $q(\tilde{z})$ are not guaranteed to be finite. However, in the important case of a normally distributed random variable $\tilde{z}, p(\tilde{z})$ and $q(\tilde{z})$ are finite, and equal the standard deviation.

In order to apply the framework from Chen et al. (2007), we first represent the uncertain parameters in each set of constraints in terms of independent factors 
$\tilde{z}^{\alpha} \in \Re^{G^{\alpha}}, \tilde{z}_{t}^{\rho} \in \Re^{G_{t}^{\rho}}$ and $\tilde{z}_{t}^{\mu} \in \Re^{G_{t}^{\mu}}$ with zero means. Let $\boldsymbol{\Xi}^{\alpha}, \boldsymbol{\Xi}_{t}^{\rho}$, and $\boldsymbol{\Xi}_{t}^{\mu}$ be the covariance matrices for the vectors $\tilde{\boldsymbol{\alpha}}, \tilde{\boldsymbol{\rho}}_{t}$, and $\tilde{\boldsymbol{\mu}}_{t}$, respectively. We assume that the vectors of uncertain coefficients $\tilde{\boldsymbol{\alpha}}, \tilde{\boldsymbol{\rho}}_{t}$, and $\tilde{\boldsymbol{\mu}}_{t}$ can be defined in terms of the independent factors $\tilde{\boldsymbol{z}}^{\alpha}, \tilde{\boldsymbol{z}}_{t}^{\rho}$ and $\tilde{\boldsymbol{z}}_{t}^{\mu}$ as follows:

$$
\begin{gathered}
\tilde{\boldsymbol{\alpha}}=\hat{\boldsymbol{\alpha}}+\left(\boldsymbol{\Xi}^{\alpha}\right)^{\frac{1}{2}} \cdot \tilde{\boldsymbol{z}}^{\alpha}, \\
\tilde{\boldsymbol{\rho}}_{t}=\hat{\boldsymbol{\rho}}_{t}+\left(\boldsymbol{\Xi}_{t}^{\rho}\right)^{\frac{1}{2}} \cdot \tilde{\boldsymbol{z}}_{t}^{\rho}, \\
\tilde{\boldsymbol{\mu}}_{t}=\hat{\boldsymbol{\mu}}_{t}+\left(\boldsymbol{\Xi}_{t}^{\mu}\right)^{\frac{1}{2}} \cdot \tilde{\boldsymbol{z}}_{t}^{\mu} .
\end{gathered}
$$

Let $\boldsymbol{P}^{o}, \boldsymbol{P}_{t}^{h}$, and $\boldsymbol{P}_{t}^{f}$ be the diagonal matrices with backward deviations and $\boldsymbol{Q}^{o}, \boldsymbol{Q}_{t}^{h}$, and $\boldsymbol{Q}_{t}^{f}$ be the diagonal matrices with forward deviations for factors $\tilde{\boldsymbol{z}}^{\alpha}$, $\tilde{\boldsymbol{z}}_{t}^{\rho}$ and $\tilde{\boldsymbol{z}}_{t}^{\mu}$, respectively. The asymmetric uncertainty sets for the uncertain factors $\tilde{\boldsymbol{z}}^{\alpha}, \tilde{\boldsymbol{z}}_{t}^{\rho}$ and $\tilde{\boldsymbol{z}}_{t}^{\mu}$ for $t=1, \ldots, T$ are specified as

$$
\begin{aligned}
\mathcal{A} \mathcal{U}^{o}= & \left\{z^{\alpha}: \exists \mathbf{v}^{\mathbf{o}}, \mathbf{w}^{\mathbf{o}} \in R_{+}^{M+1}, z^{\alpha}=\mathbf{v}^{\mathbf{o}}-\mathbf{w}^{\mathbf{o}},\left\|\left(\mathbf{P}^{\mathbf{o}}\right)^{-1} \mathbf{v}^{\mathbf{o}}+\left(\mathbf{Q}^{\mathbf{o}}\right)^{-1} \mathbf{w}^{\mathbf{o}}\right\|_{2} \leq \Omega^{o},\right. \\
& \left.\underline{\mathbf{z}}^{\alpha} \leq \tilde{\mathbf{z}}^{\alpha} \leq \overline{\mathbf{z}}^{\alpha}\right\}, \\
\mathcal{A} \mathcal{U}_{t}^{h}= & \left\{z_{t}^{\rho}: \exists \mathbf{v}_{\mathbf{t}}^{\mathbf{h}}, \mathbf{w}_{\mathbf{t}}^{\mathbf{h}} \in R_{+}^{2 M+1}, z_{t}^{\rho}=\mathbf{v}_{\mathbf{t}}^{\mathbf{h}}-\mathbf{w}_{\mathbf{t}}^{\mathbf{h}},\left\|\left(\mathbf{P}_{\mathbf{t}}^{\mathbf{h}}\right)^{-1} \mathbf{v}_{\mathbf{t}}^{\mathbf{h}}+\left(\mathbf{Q}_{\mathbf{t}}^{\mathbf{f}}\right)^{-1} \mathbf{w}_{\mathbf{t}}^{\mathbf{h}}\right\|_{2} \leq \Omega_{t}^{h},\right. \\
& \left.\underline{\mathbf{z}}_{t}^{\rho} \leq \tilde{\mathbf{z}}_{t}^{\rho} \leq \overline{\mathbf{z}}_{t}^{\rho}\right\}, \text { for } t=1, \ldots, T ; \text { and } \\
\mathcal{A} \mathcal{U}_{t}^{f}= & \left\{z_{t}^{\mu}: \exists \mathbf{v}_{\mathbf{t}}^{\mathbf{f}}, \mathbf{w}_{\mathbf{t}}^{\mathbf{f}} \in R_{+}^{M+T-t+2}, z_{t}^{\mu}=\mathbf{v}_{\mathbf{t}}^{\mathbf{f}}-\mathbf{w}_{\mathbf{t}}^{\mathbf{f}},\left\|\left(\mathbf{P}_{\mathbf{t}}^{\mathbf{f}}\right)^{-1} \mathbf{v}_{\mathbf{t}}^{\mathbf{f}}+\left(\mathbf{Q}_{\mathbf{t}}^{\mathbf{f}}\right)^{-1} \mathbf{w}_{\mathbf{t}}^{\mathbf{f}}\right\|_{2} \leq \Omega_{t}^{f},\right. \\
& \left.\underline{\mathbf{z}}_{t}^{\mu} \leq \tilde{\mathbf{z}}_{t}^{\mu} \leq \overline{\mathbf{z}}_{t}^{\mu}\right\}, \quad \text { for } t=1, \ldots, T-1 .
\end{aligned}
$$

Given the representation of the uncertain coefficients as linear combinations of factors, the constraints can be written in bilinear form. For example, the constraint

$$
\tilde{\boldsymbol{\alpha}}^{\prime} \epsilon-\phi-P-v \geq 0
$$

can be written in terms of the uncertain factors $\tilde{z}^{\alpha}$ as

$$
\tilde{\boldsymbol{\alpha}}^{\prime} \boldsymbol{\epsilon}-\phi-P-v=\hat{\boldsymbol{\alpha}}^{\prime} \boldsymbol{\epsilon}-\phi-P-v+\sum_{j=1}^{M+1} \boldsymbol{e}_{j}^{\prime}\left(\left(\boldsymbol{\Xi}^{\alpha}\right)^{\frac{1}{2}}\right)^{\prime} \boldsymbol{\epsilon} \cdot \tilde{z}_{j}^{\alpha},
$$

where $\boldsymbol{e}_{j}$ is a vector of zeros of appropriate dimension with 1 in the $j$-th position. The robust counterpart of the constraint $\tilde{\boldsymbol{\alpha}}^{\prime} \epsilon-\phi-P-v \geq 0$ when the uncertain factors $\tilde{\boldsymbol{z}}^{\alpha}$ vary in uncertainty set $\mathcal{A} \mathcal{U}^{o}$ is

$$
\min _{\tilde{z}^{\alpha} \in \mathcal{A} \mathcal{U}^{o}}\left\{\tilde{\boldsymbol{\alpha}}^{\prime} \boldsymbol{\epsilon}\right\}-\phi-P-v \geq 0
$$


Applying Proposition 3 from Chen et al. (2007), the robust counterpart of the corresponding constraint can be represented by the following set of constraints:

$$
\begin{aligned}
\hat{\boldsymbol{\alpha}}^{\prime} \boldsymbol{\epsilon}-\phi-P-v & \geq \Omega^{o}\left\|\boldsymbol{u}^{\alpha}\right\|_{2}+\left(\boldsymbol{r}^{\alpha}\right)^{\prime}\left(\bar{z}^{\alpha}\right)+\left(\boldsymbol{s}^{\alpha}\right)^{\prime}\left(\underline{z}^{\alpha}\right) \\
u_{j}^{\alpha} & \geq-p_{j}^{o}\left(\boldsymbol{e}_{j}^{\prime}\left(\left(\boldsymbol{\Xi}^{\alpha}\right)^{\frac{1}{2}}\right)^{\prime} \boldsymbol{\epsilon}+r_{j}^{\alpha}-s_{j}^{\alpha}\right), \quad j=1, \ldots, M+1 \\
u_{j}^{\alpha} & \geq q_{j}^{o}\left(\boldsymbol{e}_{j}^{\prime}\left(\left(\boldsymbol{\Xi}^{\alpha}\right)^{\frac{1}{2}}\right)^{\prime} \boldsymbol{\epsilon}+r_{j}^{\alpha}-s_{j}^{\alpha}\right), \quad j=1, \ldots, M+1 \\
\boldsymbol{r}^{\alpha}, \boldsymbol{s}^{\alpha} & \geq 0
\end{aligned}
$$

The robust counterpart of the constraint

$$
\phi-\min _{\tilde{z}^{\alpha} \in \mathcal{A} \mathcal{U}^{o}}\left\{\kappa\left(\tilde{\boldsymbol{\alpha}}^{\prime} \boldsymbol{\epsilon}-P\right)\right\} \geq 0
$$

involves the same type of constraints plus

$$
\phi-\kappa\left(\hat{\boldsymbol{\alpha}}^{\prime} \boldsymbol{\epsilon}-P\right) \geq \kappa\left(\Omega^{o}\left\|\boldsymbol{u}^{\alpha}\right\|_{2}+\left(\boldsymbol{r}^{\alpha}\right)^{\prime} \bar{z}^{\alpha}+\left(\boldsymbol{s}^{\alpha}\right)^{\prime} \underline{z}^{\alpha}\right)
$$

Next, we extend the same derivation to the set of constraints

$$
\xi_{t}^{0} \leq \xi_{t-1}^{0}+\min _{\tilde{\boldsymbol{\rho}}_{t} \in \mathcal{A} \mathcal{U}_{t}^{h}}\left\{\tilde{\boldsymbol{\rho}}_{t} \cdot \boldsymbol{\pi}_{t}\right\}
$$

for $t=1, \ldots, T$ to find their robust counterparts. The following constraints are reinjected into the robust model:

$$
\begin{aligned}
& \xi_{t}^{0} \leq \xi_{t-1}^{0}+\hat{\boldsymbol{\rho}}_{t}^{\prime} \boldsymbol{\pi}_{t}-\Omega_{t}^{h}\left\|\boldsymbol{u}_{t}^{h}\right\|_{2}-\left(\boldsymbol{r}_{t}^{\rho}\right)^{\prime} \overline{\boldsymbol{z}}_{t}^{\rho}-\left(\boldsymbol{s}_{t}^{\rho}\right)^{\prime} \underline{\boldsymbol{z}}_{t}^{\rho}, \quad t=1, \ldots, T \\
& u_{t, j}^{\rho} \geq-p_{j}^{\rho}\left(\boldsymbol{e}_{j}^{\prime}\left(\left(\boldsymbol{\Xi}_{t}^{\rho}\right)^{\frac{1}{2}}\right)^{\prime} \boldsymbol{\pi}_{t}+r_{t, j}^{\rho}-s_{t, j}^{\rho}\right), \quad t=1, \ldots, T, j=1, \ldots, 2 M+1 \\
& u_{t, j}^{\rho} \geq q_{j}^{\rho}\left(\boldsymbol{e}_{j}^{\prime}\left(\left(\boldsymbol{\Xi}_{t}^{\rho}\right)^{\frac{1}{2}}\right)^{\prime} \boldsymbol{\pi}_{t}+r_{t, j}^{\rho}-s_{t, j}^{\rho}\right), \quad t=1, \ldots, T, j=1, \ldots, 2 M+1 \\
& \boldsymbol{r}_{t}^{\rho}, \boldsymbol{s}_{t}^{\rho} \geq 0
\end{aligned}
$$

Finally, we apply the same procedure to the set of constraints

$$
\min _{\tilde{\boldsymbol{\mu}}_{t} \in \mathcal{A} \mathcal{U}_{t}^{f}}\left\{\tilde{\boldsymbol{\mu}}_{t} \cdot \boldsymbol{\tau}_{t}\right\} \geq 0, \quad \text { for } t=1, \ldots, T-1
$$

The robust counterpart of $\left(\mathcal{P}^{\operatorname{rob}(R)}\right)$ under asymmetric uncertainty sets $\mathcal{A} \mathcal{U}^{o}, \mathcal{A} \mathcal{U}_{t}^{h}$ and $\mathcal{A U}_{t}^{f}$, can be written in a compact form as follows: 


$$
\begin{aligned}
& \left(\mathcal{P}^{\text {asym }}\right) \text { : } \\
& \max _{\boldsymbol{\epsilon}, \boldsymbol{\pi}_{t}, \boldsymbol{\tau}_{t}, v, \phi, \boldsymbol{w}} v \\
& \text { s.t. } \\
& \hat{\boldsymbol{\alpha}}^{\prime} \boldsymbol{\epsilon}-\phi-P-v \geq \Omega^{o}\left\|\boldsymbol{u}^{\alpha}\right\|_{2}+\left(\boldsymbol{r}^{\alpha}\right)^{\prime} \bar{z}^{\alpha}+\left(\boldsymbol{s}^{\alpha}\right)^{\prime} \underline{z}^{\alpha} \\
& u_{j}^{\alpha} \geq-p_{j}^{\alpha}\left(\boldsymbol{e}_{j}^{\prime}\left(\left(\boldsymbol{\Xi}^{\alpha}\right)^{\frac{1}{2}}\right)^{\prime} \boldsymbol{\epsilon}+r_{j}^{\alpha}-s_{j}^{\alpha}\right), \quad j=1, \ldots, M+1 \\
& u_{j}^{\alpha} \geq q_{j}^{\alpha}\left(\boldsymbol{e}_{j}^{\prime}\left(\left(\boldsymbol{\Xi}^{\alpha}\right)^{\frac{1}{2}}\right)^{\prime} \boldsymbol{\epsilon}+r_{j}^{\alpha}-s_{j}^{\alpha}\right), \quad j=1, \ldots, M+1 \\
& \hat{\boldsymbol{\alpha}}^{\prime} \boldsymbol{\epsilon}+\phi+\kappa P \geq \kappa\left(\Omega^{o}\left\|\boldsymbol{u}^{\alpha}\right\|_{2}+\left(\boldsymbol{r}^{\alpha}\right)^{\prime} \bar{z}^{\alpha}+\left(\boldsymbol{s}^{\alpha}\right)^{\prime} \underline{z}^{\alpha}\right) \\
& \boldsymbol{r}^{\alpha}, \boldsymbol{s}^{\alpha} \geq 0 \\
& \xi_{t}^{m}=\xi_{t-1}^{m}-\eta_{t}^{m}+\zeta_{t}^{m}, \quad t=1, \ldots, T, m=1, \ldots, M \\
& \xi_{t}^{0} \leq \xi_{t-1}^{0}+\hat{\boldsymbol{\rho}}_{t}^{\prime} \boldsymbol{\pi}_{t}-\Omega_{t}^{h}\left\|\boldsymbol{u}_{t}^{h}\right\|_{2}-\left(\boldsymbol{r}_{t}^{\rho}\right)^{\prime} \bar{z}_{t}^{\rho}-\left(\boldsymbol{s}_{t}^{\rho}\right)^{\prime} \underline{z}_{t}^{\rho}, \quad t=1, \ldots, T \\
& u_{t, j}^{\rho} \geq-p_{j}^{\rho}\left(\boldsymbol{e}_{j}^{\prime}\left(\left(\boldsymbol{\Xi}_{t}^{\rho}\right)^{\frac{1}{2}}\right)^{\prime} \boldsymbol{\pi}_{t}+r_{t, j}^{\rho}-s_{t, j}^{\rho}\right), \quad t=1, \ldots, T, j=1, \ldots, 2 M+1 \\
& u_{t, j}^{\rho} \geq q_{j}^{\rho}\left(\boldsymbol{e}_{j}^{\prime}\left(\left(\boldsymbol{\Xi}_{t}^{\rho}\right)^{\frac{1}{2}}\right)^{\prime} \boldsymbol{\pi}_{t}+r_{t, j}^{\rho}-s_{t, j}^{\rho}\right), \quad t=1, \ldots, T, j=1, \ldots, 2 M+1 \\
& 0 \leq \hat{\boldsymbol{\mu}}_{t}^{\prime} \boldsymbol{\tau}_{t}-\Omega_{t}^{f}\left\|\boldsymbol{u}_{t}^{\mu}\right\|_{2}-\left(\boldsymbol{r}_{t}^{\mu}\right)^{\prime} \bar{z}_{t}^{\mu}-\left(\boldsymbol{s}_{t}^{\mu}\right)^{\prime} \underline{z}_{t}^{\mu}, \quad t=1, \ldots, T-1 \\
& u_{t, j}^{\mu} \geq-p_{j}^{\mu}\left(\boldsymbol{e}_{j}^{\prime}\left(\left(\boldsymbol{\Xi}_{t}^{\mu}\right)^{\frac{1}{2}}\right)^{\prime} \boldsymbol{\tau}_{t}+r_{t, j}^{\mu}-s_{t, j}^{\mu}\right), \quad t=1, \ldots, T, j=1, \ldots, M+T-t+2 \\
& u_{t, j}^{\mu} \geq q_{j}^{\mu}\left(\boldsymbol{e}_{j}^{\prime}\left(\left(\boldsymbol{\Xi}_{t}^{\mu}\right)^{\frac{1}{2}}\right)^{\prime} \boldsymbol{\tau}_{t}+r_{t, j}^{\mu}-s_{t, j}^{\mu}\right), \quad t=1, \ldots, T, j=1, \ldots, M+T-t+2 \\
& \boldsymbol{r}_{t}^{\rho}, \boldsymbol{s}_{t}^{\rho}, \boldsymbol{r}_{t}^{\mu}, \boldsymbol{s}_{t}^{\mu} \geq 0 \\
& \xi_{t}^{m} \geq 0 \text {, } \\
& t=1, \ldots, T \\
& t=1, \ldots, T, m=0, \ldots, M \\
& \eta_{t}^{m} \geq 0, \zeta_{t}^{m} \geq 0 \text {, } \\
& t=1, \ldots, T-1, m=1, \ldots, M
\end{aligned}
$$

where $\boldsymbol{w}$ consists of all the new variables $\left(\boldsymbol{u}^{\alpha}, \boldsymbol{r}^{\alpha}, \boldsymbol{s}^{\alpha}, \boldsymbol{u}_{t}^{\rho}, \boldsymbol{r}_{t}^{\rho}, \boldsymbol{s}_{t}^{\rho}, \boldsymbol{u}_{t}^{\mu}, \boldsymbol{r}_{t}^{\mu}, \boldsymbol{s}_{t}^{\mu}\right.$ for $t=1, \ldots, T)$ that are introduced for the robust counterpart to the original ALM problem $\left(\mathcal{P}^{\text {asym }}\right)$. This is also a tractable optimization problem; however, the number of decision variables and constraints is larger due to the nature of the asymmetric uncertainty set.

\section{Implementation}

This section discusses practical aspects of the implementation of the robust ALM models for investment products with guarantees. We also explain the design of a series of computational experiments in Sect. 6 to study the performance of the optimal strategies from the robust formulations. These experiments aim to show how the model parameters and the choice of uncertainty sets affect the robust investment strategy.

The robust optimization strategy, abbreviated as $\mathrm{R}$, is obtained by solving the robust counterpart of $\left(\mathcal{P}^{\operatorname{rob}(R)}\right)$ for symmetric and asymmetric uncertainty sets for different 
values of the price of robustness. For this strategy, the robustness budget parameters (i.e., $\theta^{o}, \theta_{t}^{h}, \theta_{t}^{f}$ for symmetric uncertainty sets and $\Omega^{o}, \Omega_{t}^{h}, \Omega_{t}^{f}$ for the asymmetric uncertainty sets at time $t$ ) associated with the objective function and the constraints containing uncertain coefficients are fixed.

The computational performance of the robust optimization strategy is compared with the performance of a nominal (expected value) strategy and a stochastic programming investment strategy. The nominal strategy, abbreviated as $N$, calculates the optimal investment strategy assuming that all uncertain coefficients in the optimization problem $\left(\mathcal{P}^{\operatorname{rob}(R)}\right)$ are at their expected values. This strategy is equivalent to the robust strategy when the price of robustness is zero. In this case, the optimization problem formulation is a deterministic problem solved by a risk-neutral investor, and is only used as a benchmark.

The stochastic programming strategy, abbreviated as S, maximizes the expected value of the objective function over the generated scenarios.

The simulation experiments use a rolling horizon optimization procedure that involves $T$ iterations. At each iteration, a set of $S$ scenarios for each length of time period is generated and the input parameters for the multi-period optimization problems are estimated. The optimization problem with new input parameters is solved and the first step recommended by the optimal strategy is taken. Actual realizations of the returns with respect to the predefined market structure are simulated again. These generated returns are used to compute the realized performance of the strategy and the first time period holdings are updated. The next iteration follows the same steps with reduced time horizon for the optimization models. This procedure is repeated in the same manner until the last time period. The portfolio positions at the last period represent the final realized wealth. We also consider an alternative approach, called fixed-horizon strategy, for the simulation experiments. This procedure applies the same investment strategy (obtained by solving the ALM optimization model) at the beginning of the planning horizon to evaluate with a number of future realisations.

All models are implemented in Matlab and solved with YALMIP (Löfberg 2004). The computational experiments are run on a Macbook pro $2.6 \mathrm{GHz} \mathrm{CPU}$ and $16 \mathrm{~Gb}$ of RAM.

\subsection{Data}

We consider generated and real market data for the computational experiments. For all experiments, investment decisions for the portfolio allocation are made at discrete time periods $t=0,1,2,3$. The portfolio is redeemed at the end of the investment horizon, $T=4$.

The generated data set is simulated for 10,20 and 30 risky assets and one risk-free asset using the factor model described in Ben-Tal et al. (2000). Specifically, the returns of the risky assets and the risk-free asset are computed as

$$
\begin{aligned}
\ln \left(1+r_{t}^{m}\right) & =\boldsymbol{\beta}_{m}^{\prime}\left[\delta \cdot \boldsymbol{e}+\sigma \cdot \boldsymbol{v}_{t}\right], \quad t=0,1, \ldots, N-1, m=1, \ldots, M \\
\ln \left(1+r_{t}^{0}\right) & =\delta, \quad t=0,1, \ldots, N-1
\end{aligned}
$$


Table 2 Statistical summary of the historical data (returns for each period)

\begin{tabular}{lllll}
\hline & $t=0$ & $t=1$ & $t=2$ & $t=3$ \\
\hline Mean return & & & & \\
\hline Risky asset & 0.029 & 0.051 & -0.003 & 0.005 \\
Risk-free asset & 0.014 & 0.012 & 0.007 & 0.005 \\
\hline Standard deviation & & & & \\
\hline Risky asset & 0.054 & 0.069 & 0.082 & 0.095 \\
Risk-free asset & 0.005 & 0.001 & 0.004 & 0.005 \\
\hline$p$ : Forward deviation & & & & \\
Risky asset & 0.054 & 0.070 & 0.082 & 0.095 \\
Risk-free asset & 0.005 & 0.001 & 0.005 & 0.005 \\
\hline$q$ : Backward deviation & & & & \\
\hline Risky asset & 0.070 & 0.069 & 0.085 & 0.101 \\
Risk-free asset & 0.005 & 0.002 & 0.004 & 0.005 \\
\hline
\end{tabular}

where $\boldsymbol{v}_{0}, \boldsymbol{v}_{1}, \ldots, \boldsymbol{v}_{N-1}$ are independent $k$-dimensional Gaussian random vectors with zero mean and the unit covariance matrix (the identity matrix). In addition, $\boldsymbol{e} \in \mathfrak{R}^{K}=(1, \ldots, 1)^{\prime} ; \boldsymbol{\beta}_{m} \in \mathfrak{R}_{+}^{K}$ are fixed vectors; and $\delta, \sigma>0$ are fixed reals. Using this model, the expected values and the covariances of the cumulative returns at time $t$ can be computed in closed form. All simulation parameters are selected as in Ben-Tal et al. (2000).

The real market data set is obtained from Goyal and Welch (2008) and consists of two assets: the S\&P 500 index and a Treasury bill. The only reason for selecting a small number of assets for investment and a short investment horizon is to illustrate specific characteristics of various investment strategies. A sample period of 24 years of quarterly data between 1987 and 2010 is considered to generate the scenarios for $\boldsymbol{R}_{t}$ as well as the cumulative risk-free rate $R_{t}^{0}$ at each time period $t$. The historical data with quarterly prices over 24 years is divided into four time periods. The mean cumulative return of each asset at each time period is estimated using the corresponding data set. The estimated expected values of the returns and the factors, as well as other descriptive statistics, such as standard deviations and backward and forward deviations, are presented in Table 2 .

The descriptive statistics of the factors $\tilde{z}^{\alpha}, \tilde{z}_{t}^{\rho}$ and $\tilde{z}_{t}^{\mu}$ extracted from the real market data set are summarized in Table 3 . The estimation procedure is described in more detail next.

\subsection{Parameter estimation for the robust formulations}

While the input parameters to the robust formulations can be calculated in closed form for certain types of processes followed by the uncertain parameters, a practical 
Table 3 Descriptive statistics for the entries of the vectors of factors $\tilde{z}^{\alpha}$, $\tilde{z}_{t}^{\rho}$ and $\tilde{z}_{t}^{\mu}$ extracted from real data

\begin{tabular}{|c|c|c|c|c|c|c|c|}
\hline$\tilde{\boldsymbol{z}}^{\alpha}:$ & Factors & 1 & 2 & & & & \\
\hline & Std dev & 0.99906 & 0.97472 & & & & \\
\hline & $p$ : Forward dev & 1.02573 & 0.99454 & & & & \\
\hline & $q$ : Backward dev & 0.99906 & 0.97472 & & & & \\
\hline$\tilde{\boldsymbol{z}}_{t}^{\rho}:$ & Factors & 1 & 2 & 3 & & & \\
\hline \multirow[t]{3}{*}{$t=1$} & Std dev & 1.03192 & 1.05277 & 0.95624 & & & \\
\hline & $p:$ Forward dev & 1.03192 & 1.05277 & 0.95626 & & & \\
\hline & $q$ : Backward dev & 1.03398 & 1.05487 & 0.95624 & & & \\
\hline \multirow[t]{3}{*}{$t=2$} & Std dev & 0.96412 & 0.98360 & 0.95167 & & & \\
\hline & $p$ : Forward dev & 0.96428 & 0.98376 & 0.95169 & & & \\
\hline & $q$ : Backward dev & 0.96412 & 0.98360 & 0.95167 & & & \\
\hline \multirow[t]{3}{*}{$t=3$} & Std dev & 0.93665 & 0.95557 & 0.95059 & & & \\
\hline & $p:$ Forward dev & 0.93718 & 0.95611 & 0.95061 & & & \\
\hline & $q$ : Backward dev & 0.93665 & 0.95557 & 0.95059 & & & \\
\hline \multirow[t]{3}{*}{$t=4$} & Std dev & 0.91791 & 0.93646 & 0.95750 & & & \\
\hline & $p:$ Forward dev & 0.91843 & 0.93699 & 0.95752 & & & \\
\hline & $q$ : Backward dev & 0.91791 & 0.93646 & 0.95750 & & & \\
\hline$\tilde{z}_{t}^{\mu}:$ & Factors & 1 & 2 & 3 & 4 & 5 & 6 \\
\hline \multirow[t]{3}{*}{$t=1$} & Std dev & 0.99852 & 0.96851 & 1.01065 & 1.10211 & 1.14325 & 1.15469 \\
\hline & $p:$ Forward dev & 0.99852 & 0.96880 & 1.01324 & 1.10368 & 1.14500 & 1.15645 \\
\hline & $q$ : Backward dev & 1.00034 & 0.96851 & 1.01065 & 1.10211 & 1.14325 & 1.15469 \\
\hline \multirow[t]{3}{*}{$t=2$} & Std dev & 0.99897 & 0.98629 & 1.09494 & 1.13022 & 1.14152 & \\
\hline & $p:$ Forward dev & 0.99914 & 0.98708 & 1.09646 & 1.13222 & 1.14354 & \\
\hline & $q$ : Backward dev & 0.99897 & 0.98629 & 1.09494 & 1.13022 & 1.14152 & \\
\hline \multirow[t]{3}{*}{$t=3$} & Std dev & 0.99868 & 0.97976 & 1.13039 & 1.14170 & & \\
\hline & $p:$ Forward dev & 0.99923 & 0.98068 & 1.13155 & 1.14286 & & \\
\hline & $q$ : Backward dev & 1.01170 & 0.99253 & 1.14512 & 1.15657 & & \\
\hline
\end{tabular}

approach to estimating them for any assumptions on their dynamics is to generate scenarios for the realizations of the uncertain vectors $\tilde{\boldsymbol{\alpha}}, \tilde{\boldsymbol{\rho}}_{t}$ and $\tilde{\boldsymbol{\mu}}_{t}$. These scenarios can then be used to extract the necessary information. In our computational experiments, the latter scenario approach is used for generating the inputs in both the symmetric and the asymmetric uncertainty set formulation, and with both the generated and the real data set.

Symmetric uncertainty set: Suppose we have scenarios for the vectors of cumulative returns $\boldsymbol{R}_{t}$ at each time period $t$ and the cumulative risk-free rate $R_{t}^{0}$ for $t=1, \ldots, T$. They are used to create scenarios for the uncertain vectors $\left(\tilde{\boldsymbol{\alpha}}, \tilde{\boldsymbol{\rho}}_{t}\right.$ and $\left.\tilde{\boldsymbol{\mu}}_{t}\right)$ in each constraint of the optimization problem. These scenarios in turn are used to estimate the expected value vectors $\hat{\boldsymbol{\alpha}}, \hat{\boldsymbol{\rho}}_{t}$ and $\hat{\boldsymbol{\mu}}_{t}$, as well as the covariance matrices $\boldsymbol{\Xi}^{\alpha}, \boldsymbol{\Xi}_{t}^{\rho}$ 
and $\boldsymbol{\Xi}_{t}^{\mu}$ that are input parameters to the robust optimization models with symmetric uncertainty sets $\left(\mathcal{P}^{\text {sym }}\right)$.

Asymmetric uncertainty set: In the case of asymmetric uncertainty set, we need estimates of the forward and backward deviations of the factors $\tilde{z}^{\alpha}, \tilde{z}_{t}^{\rho}$, and $\tilde{z}_{t}^{\mu}$. Similarly to the case of symmetric uncertainty set, we use generated scenarios for vectors of cumulative returns $\boldsymbol{R}_{t}$ at each time period $t$ and the cumulative risk-free rate $R_{t}^{0}$ for $t=1, \ldots, T$ to create scenarios for the uncertain vectors $\tilde{\boldsymbol{\alpha}}, \tilde{\boldsymbol{\rho}}_{t}$ and $\tilde{\boldsymbol{\mu}}_{t}$ in each constraint of the optimization problem. A set of scenarios for the uncorrelated factors $\tilde{z}^{\alpha}$, $\tilde{\boldsymbol{z}}_{t}^{\rho}$, and $\tilde{\boldsymbol{z}}_{t}^{\mu}$ for each constraint can then be derived from the scenarios for $\tilde{\boldsymbol{\alpha}}, \tilde{\boldsymbol{\rho}}_{t}$ and $\tilde{\boldsymbol{\mu}}_{t}$ as

$$
\begin{aligned}
& \tilde{z}^{\alpha}=\left(\boldsymbol{\Xi}^{\alpha}\right)^{-\frac{1}{2}} \cdot(\tilde{\boldsymbol{\alpha}}-\hat{\boldsymbol{\alpha}}), \\
& \tilde{z}_{t}^{\rho}=\left(\boldsymbol{\Xi}_{t}^{\rho}\right)^{-\frac{1}{2}} \cdot\left(\tilde{\boldsymbol{\rho}}_{t}-\hat{\boldsymbol{\rho}}_{t}\right), \\
& \tilde{\boldsymbol{z}}_{t}^{\mu}=\left(\boldsymbol{\Xi}_{t}^{\mu}\right)^{-\frac{1}{2}} \cdot\left(\tilde{\boldsymbol{\mu}}_{t}-\hat{\boldsymbol{\mu}}_{t}\right) .
\end{aligned}
$$

The scenarios for $\tilde{\boldsymbol{z}}^{\alpha}, \tilde{\boldsymbol{z}}_{t}^{\rho}$, and $\tilde{\boldsymbol{z}}_{t}^{\mu}$ are used to estimate the factors' backward and forward deviations, which are then plugged into the robust ALM formulation $\left(\mathcal{P}^{\text {asym }}\right)$. For example, the backward and forward deviations for the $i$ th factor of $\tilde{z}^{\alpha}$ can be computed by solving the optimization problems

$p_{i}\left(z^{\alpha}\right)=\sup _{\varphi>0}\left\{\sqrt{2 \frac{\ln \left(E\left(\exp \left(\varphi \cdot \mathbf{z}^{\alpha}\right)\right)\right)}{\varphi^{2}}}\right\}$ and $q_{i}\left(z^{\alpha}\right)=\sup _{\varphi>0}\left\{\sqrt{2 \frac{\ln \left(E\left(\exp \left(-\varphi \cdot \mathbf{z}^{\alpha}\right)\right)\right)}{\varphi^{2}}}\right\}$

The reader is referred to Natarajan et al. (2008) for a proof of this relationship. If the forward and backward deviation matrices are equal (i.e., $p_{i}(z)=q_{i}(z)$ for all $i$ ), then the asymmetric uncertainty set robust formulation produces the same portfolio composition as the ellipsoidal uncertainty set does.

\section{Computational results}

In this section, we discuss the results of the computational experiments to illustrate the performance of the ALM investment strategies obtained with the nominal, robust optimization, and scenario-based stochastic programming models under different market conditions. Due to length constraints, we only present representative sets of computational results. In a normal market regime, we assume that the market behaves as expected. In other words, future return scenarios for evaluating performance are generated with the originally estimated mean $(\mu)$ and variance $\left(\sigma^{2}\right)$. For an unfavorable market regime, we assume that the investor invests optimally given a particular expected return, but in actuality asset returns follow a distribution that is worse than expected on average. Specifically, the future return scenarios are generated with a mean value of $(\mu-k \sigma)$ and the same estimated variance $\left(\sigma^{2}\right)$ as in the normal market conditions. The factor $k=0,25,0.5,0.75,1.0$ denotes the level of unfavorable market. Note that $k=0$ refers to the normal market regime. We evaluate the performance of the robust strategies in several ways: 
- relative to the performance of the nominal and the stochastic programming models in terms of summary statistics for the realized final wealth;

- in terms of the optimal asset allocation at the first step of the multi-period optimization problem as well as the CPU time taken to obtain an investment strategy;

- in terms of the effect of using asymmetric versus a symmetric uncertainty set in the robust formulation; and

- in terms of the effect of the magnitude of different parameters associated with the robust formulation, such as the budget of robustness.

For the numerical experiments, other inputs to the robust ALM models are selected as follows. We assume that there is no initial investment in the fund. The principal amount is $P=\$ 1000$. The guaranteed rate of return is $\bar{g}=1 \%$ per period for the real market data and $\bar{g}=5 \%$ per period for the generated data set. The principal plus some return accumulated over time are withdrawn at the end of investment horizon. We keep track only of the excess return. The participation rate $(\kappa)$ is selected as 0.5 . It is assumed that there is no coupon payment at any time period. Transactions costs for buying $\left(c_{\mathrm{b}}\right)$ and selling $\left(c_{\mathrm{s}}\right)$ are both fixed at $1 \%$. The funding ratio $(\psi)$ is set at 0.9 . The values selected for the budget of robustness for the symmetric and asymmetric uncertainty sets associated with the funding ratio and balance constraints as well as the objective function are between 0.1 and 1 .

For the stochastic programming models, a scenario tree with a fan of individual scenarios is considered. More precisely, the scenario tree consists of 100 scenarios branching in the first time period and no branching at further stages over 4 and 10 time periods. It should be emphasized that the scenario-based stochastic optimization formulation in this case is a deterministic problem, and is only used as a benchmark. On average, the investment strategy obtained by the scenario-based stochastic model will always outperform the robust decisions given the fact that it optimizes the expected wealth, while the robust strategy focuses on worst-case outcomes.

In the rolling horizon and fixed horizon simulation procedures, the optimal investment strategies are evaluated for 1000 realized future asset returns to compute the final wealth. The statistical analysis of simulated values of the final wealth is presented in terms of mean, variance, minimum and maximum out of 1000 simulations. We also compute tail risk measures reminiscent of Value-at-Risk (VaR) and Conditional Value-at-Risk (CVaR). The "VaR" at $5 \%$ is found by taking the 50th smallest realized portfolio value whereas the "CVaR" is calculated as the average of the 50 smallest portfolio values in all simulations.

With a slight abuse of terminology but for simplicity's sake, when we discuss computational results in the rest of this section we will refer to products with the more general structure defined in Sect. 2 as ELN, and to products with the more simple structure with $\kappa=0$ and fixed coupon payments as GIC.

Problem structure and asset allocation: We first illustrate the problem structure and performance characteristics of the different ALM models for ELN products in Table 4. It is worthwhile to mention that all types of ALM models for GIC and ELN show similar characteristics. The sizes of problems for the nominal, stochastic and robust optimization models are defined in terms of the number of variables and constraints. As 
Table 4 Problem structure and performance of the ALM models

\begin{tabular}{|c|c|c|c|c|c|c|c|}
\hline \multirow{2}{*}{$\begin{array}{l}\text { ALM } \\
\text { models }\end{array}$} & \multicolumn{3}{|c|}{ Problem structure } & \multirow{2}{*}{$\begin{array}{l}\text { Solution } \\
\text { time }\end{array}$} & \multirow{2}{*}{$\begin{array}{l}\text { Final } \\
\text { wealth }\end{array}$} & \multirow{2}{*}{$\begin{array}{l}\text { Transaction } \\
\text { cost }\end{array}$} & \multirow{2}{*}{$\begin{array}{l}\text { \# Assets } \\
\text { invested }\end{array}$} \\
\hline & $(T, M)$ & Variables & Constraints & & & & \\
\hline $\mathrm{N}$ & $(4,2)$ & 30 & 46 & 0.020 & 7.560 & 9.800 & 1 \\
\hline Sym-R(1) & $(4,2)$ & 62 & 70 & 0.030 & 4.011 & 9.540 & 2 \\
\hline Asym-R(1) & $(4,2)$ & 78 & 102 & 0.050 & 4.010 & 9.540 & 2 \\
\hline SP & $(4,2)$ & 3029 & 13,934 & 0.120 & 7.340 & 10.030 & 1 \\
\hline $\mathrm{N}$ & $(4,11)$ & 126 & 174 & 0.030 & 74.645 & 10.176 & 1 \\
\hline Sym-R(1) & $(4,11)$ & 222 & 262 & 0.040 & -44.046 & 7.768 & 11 \\
\hline Asym-R(1) & $(4,11)$ & 302 & 422 & 0.060 & -44.744 & 7.568 & 11 \\
\hline SP & $(4,11)$ & 12,725 & 26,038 & 0.340 & 68.546 & 9.870 & 2 \\
\hline $\mathrm{N}$ & $(4,21)$ & 246 & 334 & 0.054 & 76.106 & 10.161 & 1 \\
\hline Sym-R(1) & $(4,21)$ & 422 & 502 & 0.120 & -24.230 & 8.528 & 17 \\
\hline Asym-R(1) & $(4,21)$ & 582 & 822 & 0.491 & 8.519 & 8.519 & 17 \\
\hline SP & $(4,21)$ & 24,845 & 41,168 & 0.410 & 69.540 & 9.177 & 3 \\
\hline $\mathrm{N}$ & $(4,31)$ & 366 & 494 & 0.101 & 80.869 & 10.122 & 1 \\
\hline Sym-R(1) & $(4,31)$ & 622 & 742 & 0.233 & -15.767 & 8.526 & 24 \\
\hline Asym-R(1) & $(4,31)$ & 862 & 1222 & 0.751 & -18.417 & 8.520 & 24 \\
\hline SP & $(4,31)$ & 36,965 & 56,298 & 1.707 & 75.392 & 10.320 & 4 \\
\hline $\mathrm{N}$ & $(10,11)$ & 222 & 432 & 0.512 & 260.832 & 17.681 & 1 \\
\hline Sym-R(1) & $(10,11)$ & 462 & 652 & 0.860 & -17.359 & 9.554 & 7 \\
\hline Asym-R(1) & $(10,11)$ & 617 & 1052 & 1.342 & -30.201 & 8.393 & 8 \\
\hline SP & $(10,11)$ & 22,421 & 52,024 & 2.084 & 217.452 & 15.790 & 2 \\
\hline $\mathrm{N}$ & $(10,21)$ & 612 & 832 & 0.609 & 318.852 & 17.687 & 1 \\
\hline Sym-R(1) & $(10,21)$ & 1052 & 1252 & 0.910 & 128.918 & 16.060 & 17 \\
\hline Asym-R(1) & $(10,21)$ & 1452 & 2052 & 1.913 & 125.504 & 16.010 & 17 \\
\hline SP & $(10,21)$ & 61,811 & 91,334 & 2.365 & 295.459 & 14.054 & 2 \\
\hline $\mathrm{N}$ & $(10,31)$ & 1512 & 2432 & 1.342 & 332.553 & 11.650 & 1 \\
\hline Sym-R(1) & $(10,31)$ & 1552 & 1852 & 1.456 & 151.101 & 8.776 & 14 \\
\hline Asym-R(1) & $(10,31)$ & 2152 & 3052 & 2.726 & 134.322 & 8.541 & 14 \\
\hline SP & $(10,31)$ & 92,111 & 130,644 & 2.908 & 296.953 & 12.948 & 2 \\
\hline
\end{tabular}

mentioned before, the size of scenario based stochastic programming models depends on the investment horizon $(T)$, the number of assets $(M)$ and the branching structure of the scenario tree.

In our experiments, the stochastic programming problems can be classified as large size linear programming whereas the robust models are medium size nonlinear programming problems. The performance of the ALM models is displayed in terms of final wealth and the CPU time (in seconds) taken to solve the underlying optimization problem. It is not quite appropriate to compare the efficiency of the stochastic and 
robust optimization models because different solution algorithms are used; however, one can see that the CPU time increases when the size of the problem increases.

The nominal approach (expected value optimization) always results in the highest expected wealth compared to the stochastic programming and robust optimization models (Table 4). Table 4 also summarizes the total transaction cost paid over the investment horizon as well as the number of assets invested in the first time period. The realized transaction costs tend to be the lowest for the robust optimization approaches. This is consistent with previous observations in the literature that robust optimization portfolio allocation schemes reduce portfolio turnover (Fabozzi et al. 2007).

From the results in Table 4, we observe that the robust strategies prefer to invest in a smaller number of risky assets as the length of the investment horizon is increased from 4 to 10 time periods. The financial intuition for this behaviour could be that when the investment horizon gets longer, by virtue of being more inert and conservative robust optimization strategies are better at compensating for possible losses in some periods by not trading, so diversification is less important as a way to achieve a goal. The stochastic programming and nominal strategies do not change as much as the number of time periods changes. They also prefer to invest in fewer assets than the robust strategies, resulting in worse diversification. We should note that the topology of the scenario tree plays an important role for diversification strategies suggested by the stochastic optimization model.

Table 5 presents the optimal asset allocations (in a risky asset and a risk-free asset abbreviated as RK and RF, respectively) obtained by solving the nominal and robust models under the normal market regime for the real market data for various values of the budget of robustness. We look at the first step of the rolling horizon simulation approach (the step that is actually implemented). Note that the initial allocation of the capital between risk-free and risky assets does not remain the same at the following stages of the investment process due to updates for the future realizations of returns. The scenario-based stochastic programming ALM models for both types of products suggests investing the whole capital (952.7) in the risky asset in the first time period. The asset allocation at the next stages may be different because it depends on the future return realizations.

Based on the results in Table 5, it appears that the optimal asset allocation strategy obtained with the nominal model prefers to invest heavily in the risky asset rather than the risk-free asset at each time period. With regard to the robust strategy, the choice of uncertainty sets and the structure of the guaranteed investment contract do not have any substantial impact on diversification (i.e., the relative magnitude of investments in the risky versus the risk-free asset). The level of diversification of the robust investment strategy depends on the pre-specified price of robustness. If the price of robustness is low, the capital is invested mainly in the risky asset. As the price of robustness increases, the robust strategies become more risk-averse. Investment in the risky asset decreases while investment in the risk-free asset increases to hedge for the increase in risk associated with investing in the index. There appears to be a threshold $(0.5)$ for 


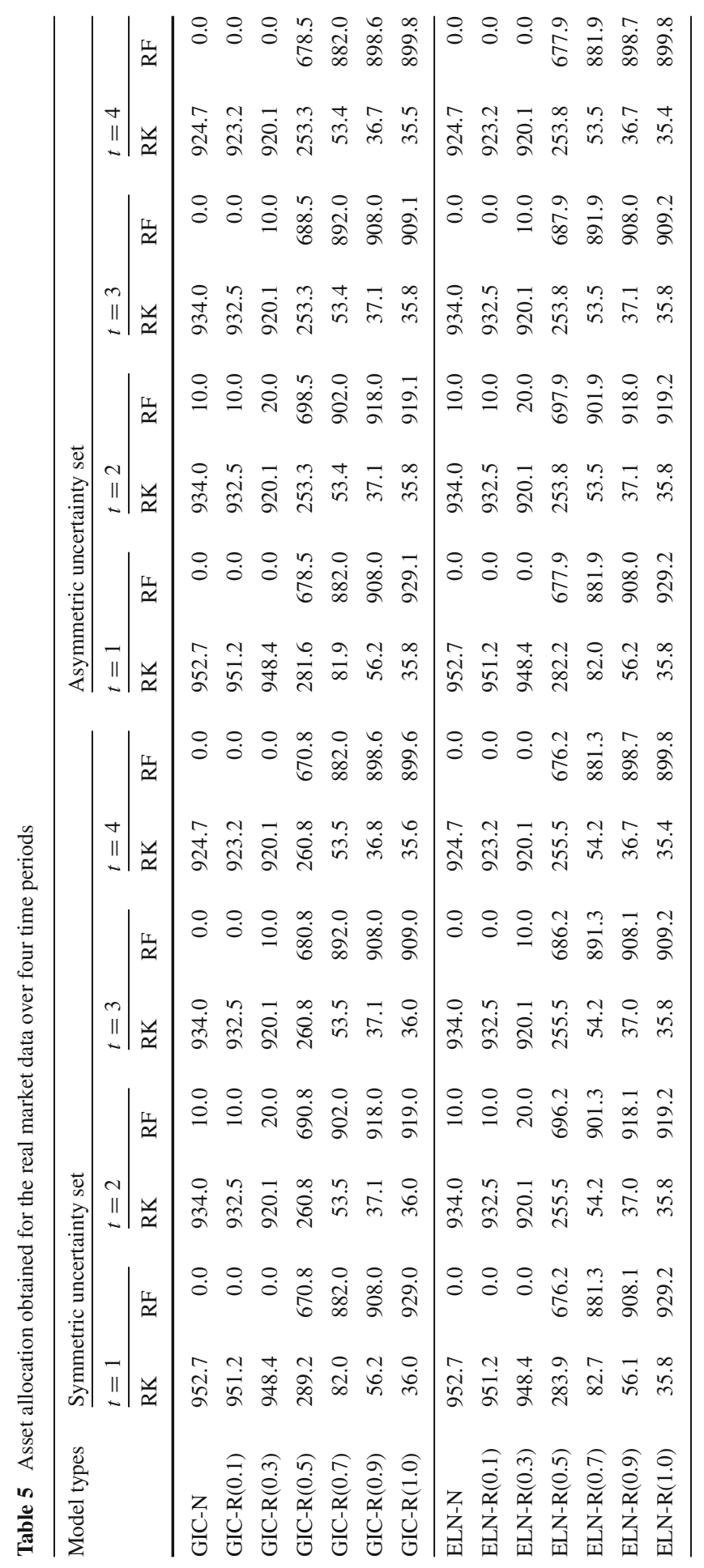


the budget of robustness. As the budget of robustness gets higher than the threshold, the weight of the risky (risk-free) asset rapidly decreases (increases).

Simulation results: We are now concerned with performance comparison of the nominal, robust and stochastic approaches under normal and unfavorable market regimes. Table 6 presents the results of the rolling horizon simulation approach using generated data with 10 risky assets and one risk-free asset within the normal (top) and unfavorable with $k=1.0$ (bottom) market regimes. In addition to the simulation statistics, we also display the average realized transaction cost (T-cost) in Table 6. From the results in Table 6 we observe that

- The investment strategies obtained from the stochastic and nominal ALM models provide higher expected wealth (as well as higher average transaction costs) than the investment strategies obtained from the robust ALM models using symmetric and asymmetric uncertainty sets under the normal market regime. This is not surprising because stochastic programming maximizes the expected profit over known discrete scenarios whereas robust optimization uses a worst-case decision-making criterion. Moreover, under normal market regime, the future return scenarios are realized as expected.

- If the future asset returns follow the same distribution as the distribution used as input to the robust formulation, then the expected terminal wealth and the variance of terminal wealth obtained by all robust models decrease when the robustness budget increases (see, for example, the results under normal market regime). In other words, there is a trade-off between the average performance and the amount of protection desired. The nominal and stochastic investment strategies provide higher average wealth than the robust strategy for a high budget of robustness.

- On the other hand, the robust investment strategies at (high) budget of robustness appear to perform better than those obtained with the stochastic and nominal formulations in unfavorable market regimes. Recall that in the unfavorable market regime, the future return realizations are generated by the expected value $\mu-k \sigma$ (that is $k$ standard deviation lower than the estimated expected value from the data) and the standard deviation $(\sigma)$. As shown in Table 6 , the terminal wealth obtained by all robust models when future realized asset returns are worse than expected increases as the robustness budget increases, and the variance of final wealth decreases. Both the nominal and the stochastic investment strategies result in lower expected wealth than the robust strategy for symmetric and asymmetric uncertainty sets at high value of the price of robustness. This argues for using robust optimization in unfavorable market scenarios.

- The robust strategies with asymmetric uncertainty sets result in slightly higher wealth (and/or lower variance) than the wealth obtained for robust strategies generated with the symmetric uncertainty sets formulation for any degree of robustness (apart from the lowest value 0.1) under unfavorable market regimes. This is because the future return realizations for the generated data have asymmetric characteristics. It appears that the distribution of various price realizations and the choice of uncertainty set impact the performance of the robust investment strategy.

- In both market regimes, the robust GIC models result in a slightly higher expected value and variance of wealth than the robust ELN models regardless of the type 


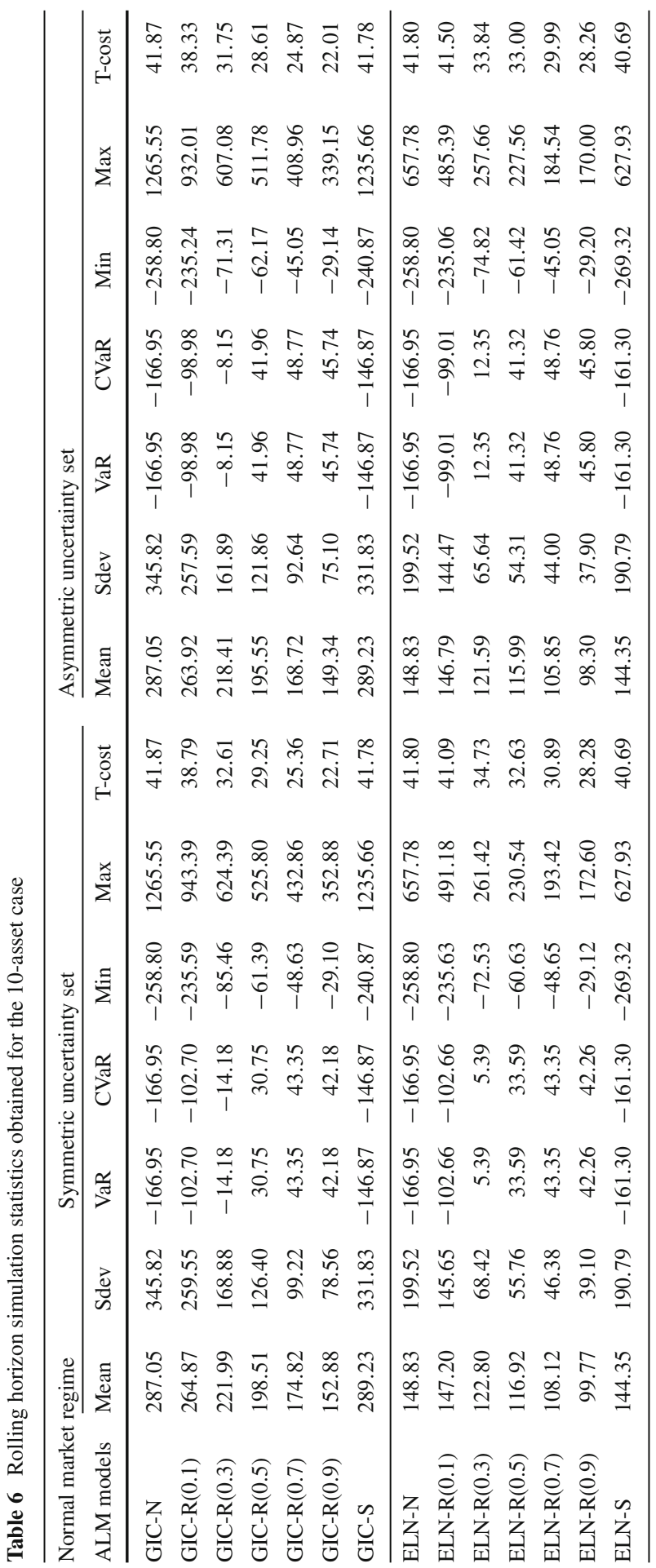




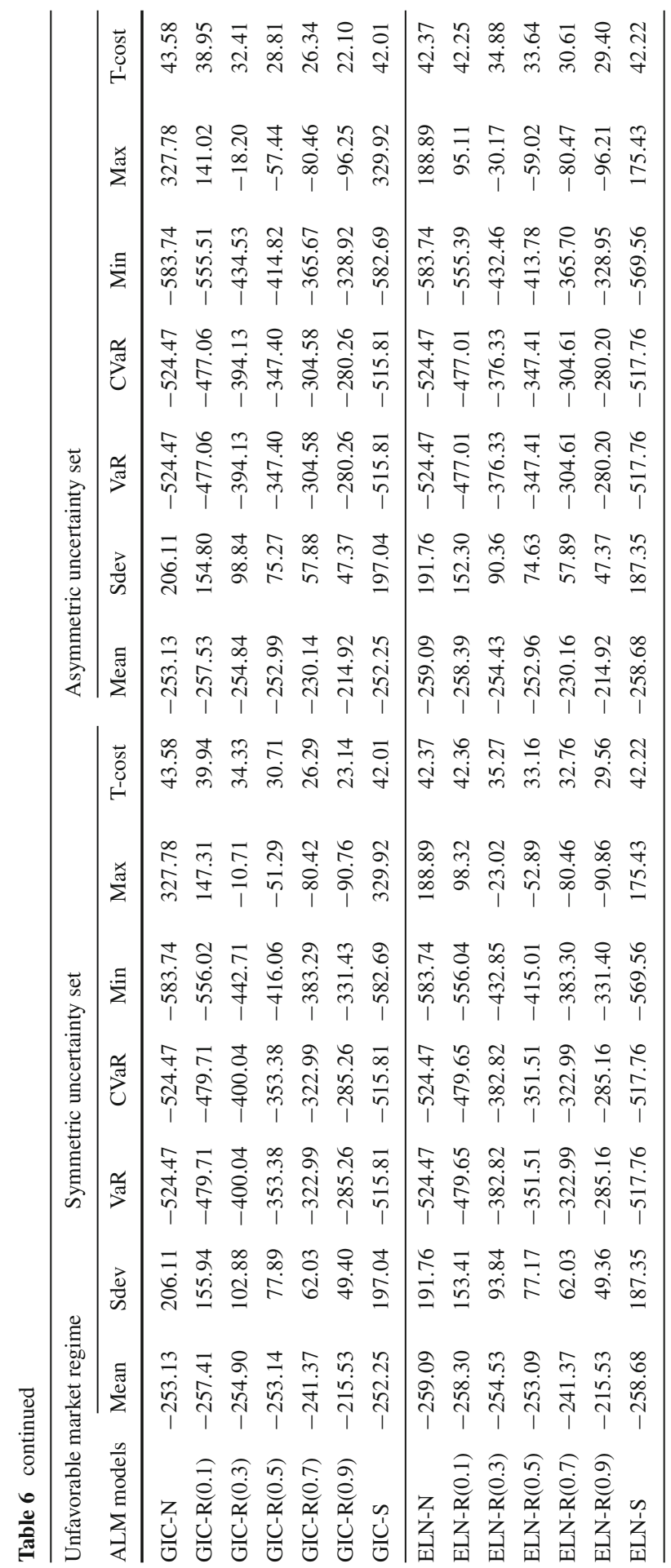



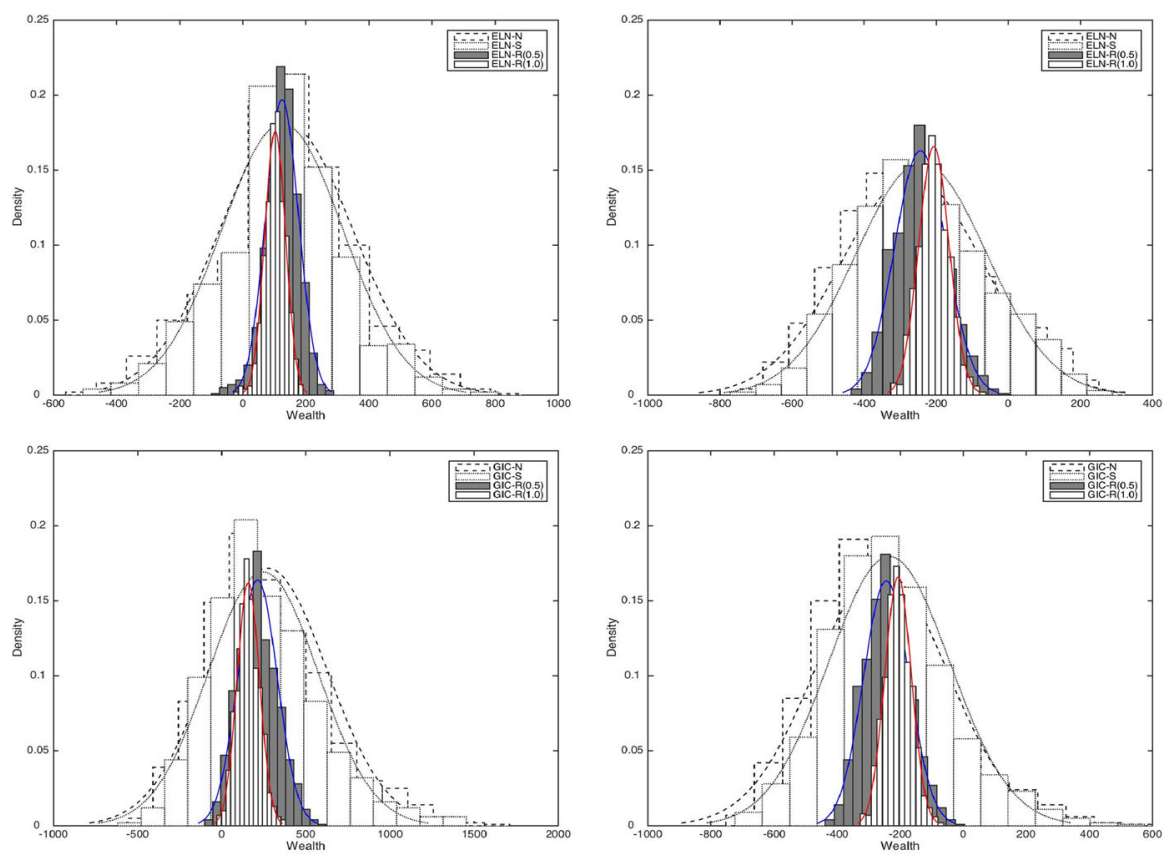

Fig. 1 Distribution of final wealth for the ALM models using ELN (top) and GIC (bottom) products in the normal (left) and unfavorable (right) market regimes. Clockwise from top left: an ELN stochastic formulation under a normal market regime; an ELN stochastic formulation under a unfavorable market regime; a GIC stochastic formulation under a unfavorable market regime; a GIC stochastic formulation under a normal market regime

of uncertainty sets. This is because the extra return on the terminal wealth is paid to the holder of ELNs. On the other hand, we observe that the ALM models for GIC products produce lower expected transaction cost than those provided by the ALM models using ELN regardless the market conditions.

Figure 1 displays the distribution of the final wealth computed from the simulation experiments using data for 10 assets with 4 time periods. In the unfavorable market regime, the future return realizations are generated with a lower mean $(\mu-\sigma)$. The histograms of final wealth outcomes for the robust optimization models with price of robustness of 0.5 and 1.0 are plotted with solid lines.

Interestingly, the relative advantage of the robust strategies increases as the market worsens - the position of the distribution of wealth under the robust strategies relative to the other two types of strategies is farther to the right. Hence, the robust approaches represent effective hedging strategies with respect to the given risk exposures (uncertainties). This can be viewed as a strong argument for adopting them in practice.

Sensitivity analysis: We also run a sensitivity analysis to determine the possible effect of the selection of model parameters on the performance of the investment strategies. We consider different market conditions where future realizations of uncertain parame- 

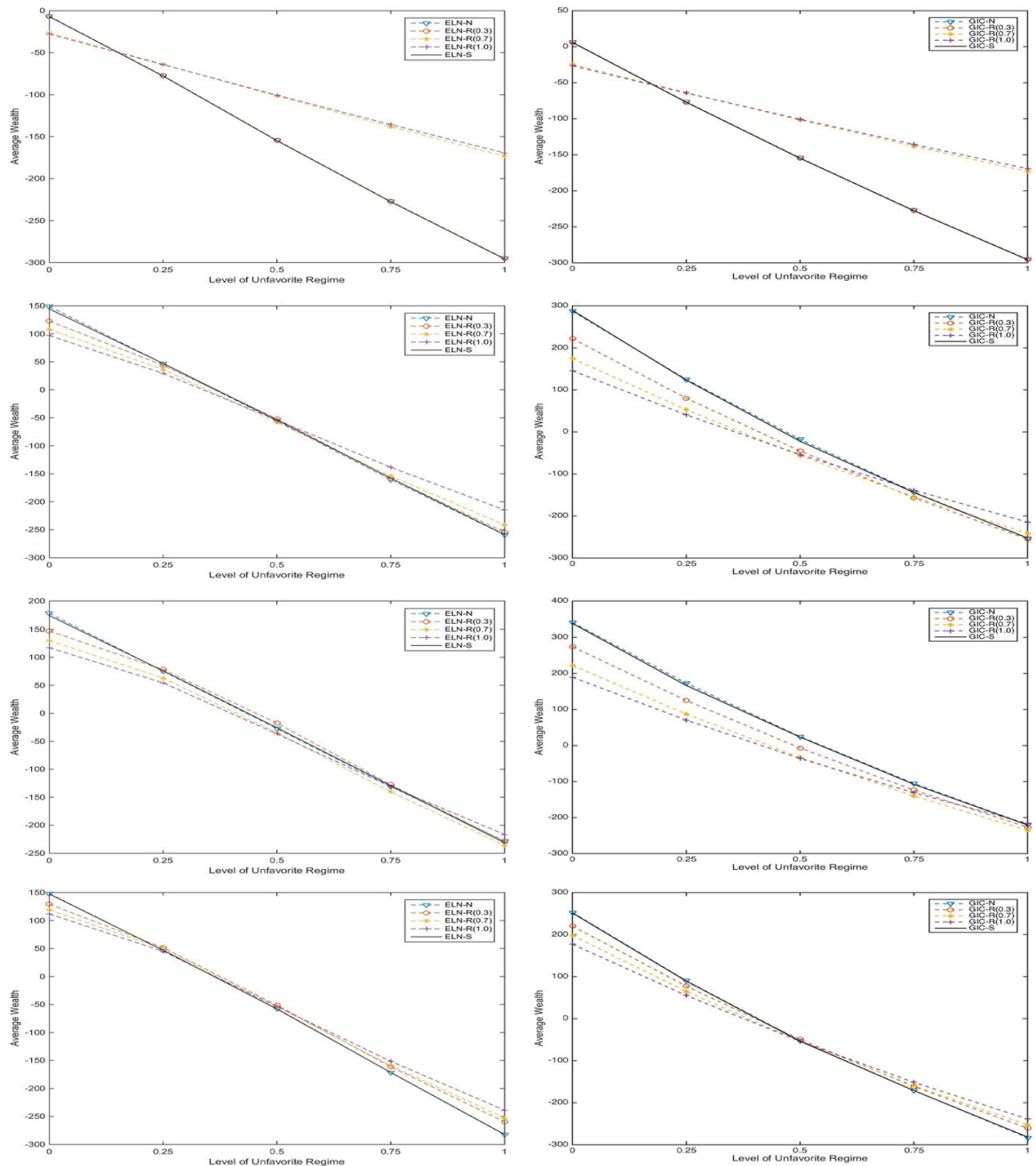

Fig. 2 Average wealth obtained for the ALM strategies for ELN (left) and GIC (right) products using 2, 10,20 and 30 (top to bottom panels) assets in various market regimes

ters are generated with different expected value $(\mu-k \sigma)$ and the same variance $(\sigma)$ as estimated from the data. As $k$ varies from 0 (normal market regime) to 1 , the expected values of the returns in future scenarios decrease. Next, we summarize observations about the effect of various parameters and factors on the ALM investment strategies obtained with the nominal, stochastic and robust optimization models.

- Number of risky assets and diversification: We first extend the simulation experiments conducted for the 2-asset real market case to a set of generated data with a larger number of assets (10, 20 and 30). The average wealth obtained with the stochastic, nominal and robust (at fixed budget of robustness of 0.3, 0.7 and 1.0) strategies is displayed in Fig. 2. As the market conditions deteriorate, the robust strategies with high budget of robustness outperform both the nominal and the 

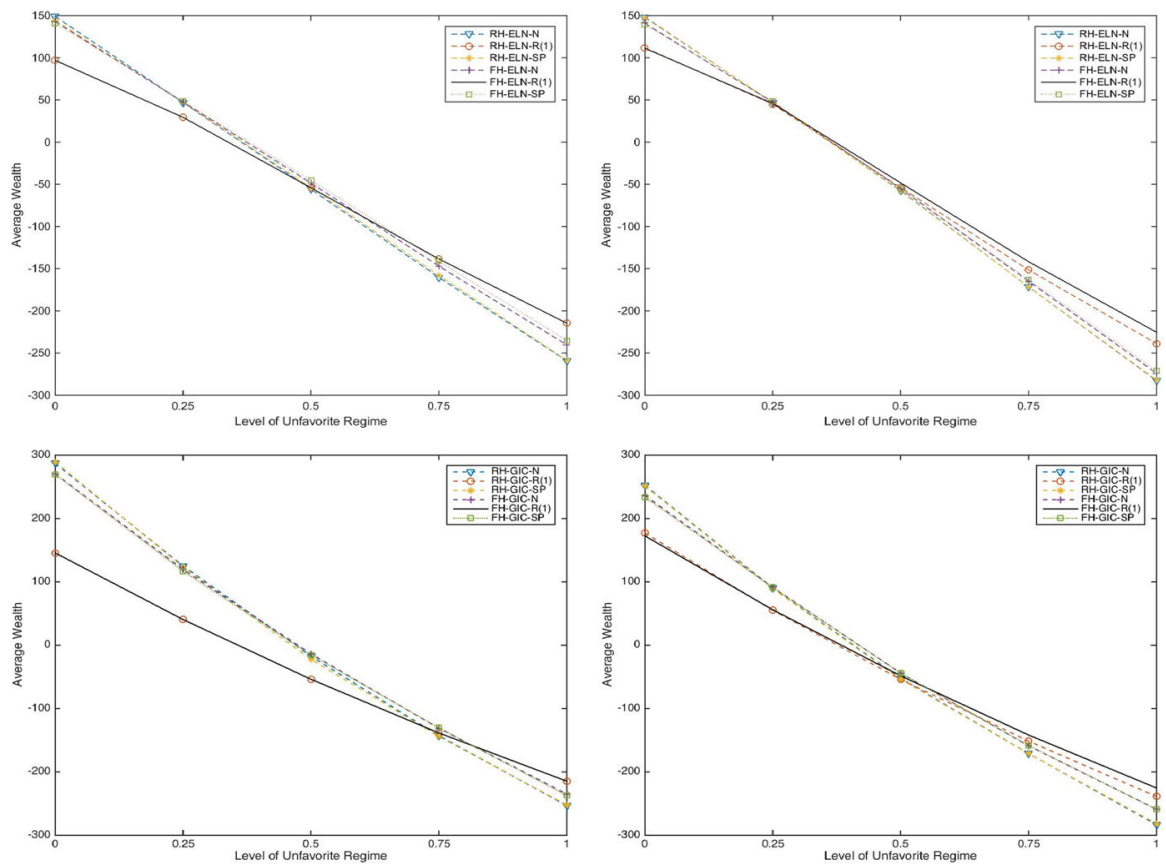

Fig. 3 Performance comparison of the ALM strategies for ELN (top) and GIC (bottom) products in various market regimes obtained by different simulation methods using 10 (left) and 30 (right) assets

stochastic ones. However, the gap between the expected wealth obtained with the stochastic programming and the robust optimization models diminishes as the number of assets increases due to the impact of diversification. Diversification appears to help the stochastic programming strategies do well even in unfavorable market conditions. For instance, while the deteriorating point for stochastic programming strategies is at $k \approx 0.15$ for the 2 -asset case, it is at $k \approx 0.5$ for the 10-asset case.

- Performance evaluation approaches: We consider static and dynamic performance evaluation approaches using cases with 10 and 30 assets. As explained before, the rolling horizon method updates the strategy as more info is received whereas the fixed horizon applies the prior strategy over time. Their results are shown in Fig. 3. We see that the average wealth obtained by the robust models is higher than the one obtained by the nominal model for smaller values of $k$ (at lower levels of the unfavorable regime) if the number of assets is large. This is because diversification has a stronger effect when there are more investment alternatives.

- Uncertainty sets: In order to understand how the size and the shape of the symmetric and asymmetric uncertainty sets affect the robust investment strategies, we vary the budget of robustness for the uncertainty sets associated with the uncertain coefficients in the objective function and the balance constraints. The numerical experiments indicate that the budget of robustness for the funding ratio constraints does not have a substantial effect on the investment decisions; hence those results on are not included here. 

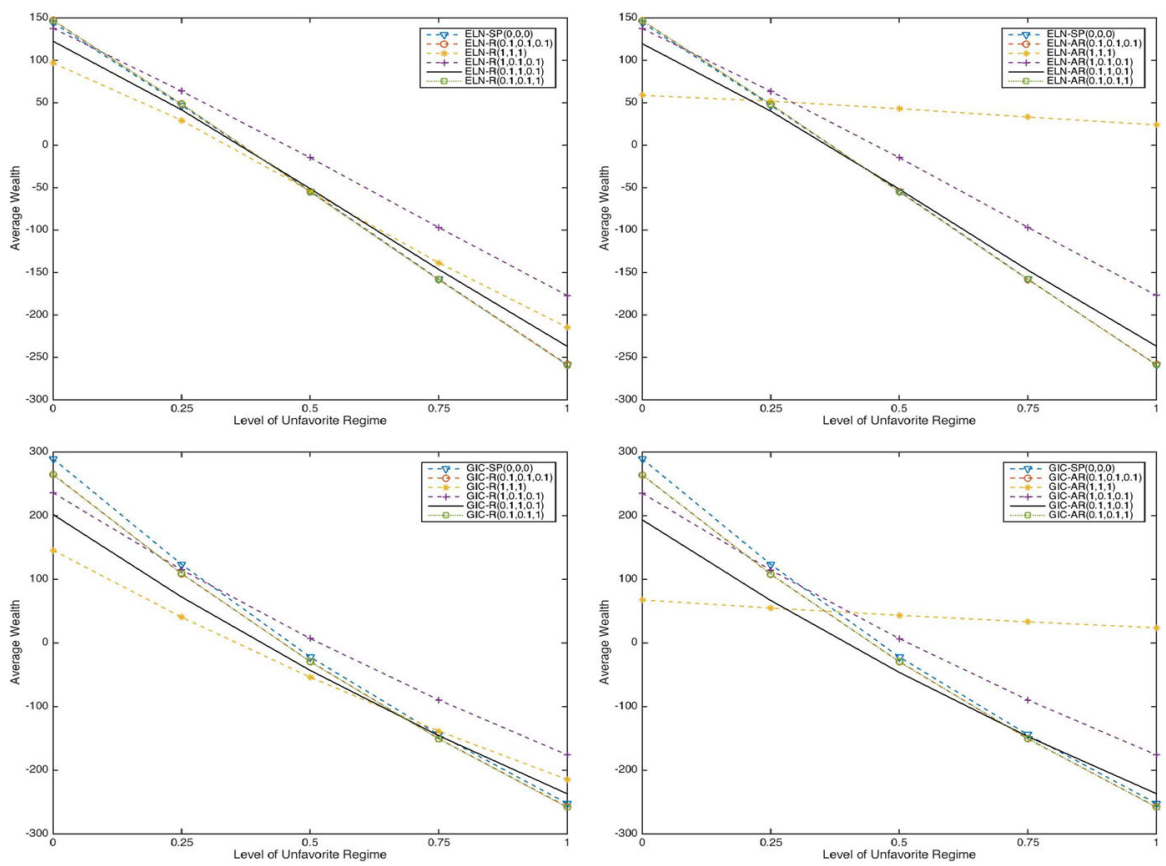

Fig. 4 Impact of the budget of robustness under various market conditions for ELN (top) and GIC (bottom) products using symmetric (left) and asymmetric (right) uncertainty sets

The simulation results presented in terms of average final wealth in Fig. 4 illustrate the effect of the budget of robustness $\left(\theta^{o}, \theta_{t}^{h}, \theta_{t}^{f}\right)$ and $\left(\Omega^{o}, \Omega_{t}^{h}, \Omega_{t}^{f}\right)$ for symmetric and asymmetric uncertainty sets, respectively, on the robust decisions under normal and unfavorable market regimes. For instance, in order to understand the impact of $\theta^{o}$, we vary $\theta^{o}$ within the interval $[0,1]$ and fix $\theta_{t}^{h}$ and $\theta_{t}^{f}$ at 0.1 . Similarly, for $\theta_{t}^{h}$ we select the same values from the interval $[0,1]$ while $\theta^{o}=\theta_{t}^{f}=0.1$. These two cases are abbreviated as $(1,0.1,0.1)$ and $(0.1,1,0.1)$, respectively, in Fig. 4. The computational results indicate that the budget of robustness (for both symmetric and asymmetric uncertainty sets) plays an important role for the performance of robust investment strategies in terms of the realized final expected wealth. The budget of robustness associated with the uncertain coefficients in the balance constraints has higher impact on the final expected wealth than the budget of robustness for the objective function. This may be because of the cumulative effect of multiple time periods. Note that there is a balance constraint at each time period whereas the objective function involves only the terminal date.

Finally, we observe that the setting of $(1,0.1,0.1)$ produces the highest expected wealth under any market regime regardless the choice of model. This may be due to the high impact of the budget of robustness (the first parameter) related to the original objective function on the final wealth. Similarly, when the budget of robustness of the asymmetric uncertainty set is fixed at $(1,1,1)$, it leads to almost the same expected wealth for any market regime for the GIC and ELN types of ALM models. 


\section{Concluding remarks}

In this paper, we considered the asset-liability management problem for investment products with guarantees where the optimal structure of the underlying fund is determined and exogenous uncertainties in asset returns and interest rates are incorporated in the model using a multi-period robust optimization framework. We introduced a tractable robust approach to solving the problem using symmetric and asymmetric uncertainty sets, and described practical approaches for estimating the inputs. The performance of the robust formulations of ALM models for investment products with guarantees were evaluated in computational experiments with simulated and real market data, contrasted with the performance of strategies based on stochastic programming, and benchmarked against the performance of expected value optimization. The robust investment strategies have better performance in terms of variability and relative return under unfavorable market regimes where the future realizations follow probability distributions with lower means than expected. The numerical results also show that the robust model parameters and the size of the uncertainty sets play an important role for the performance of robust optimization models.

When deciding on the approach for determining the optimal structure of portfolios underlying investment products with guarantees, one should note that there are philosophical differences between the application of the robust optimization approach and the stochastic programming approach. First, the representation of the uncertainties is done differently. In the stochastic programming approach, one solves the optimization problem to come up with an exact strategy to be followed for each scenario. One expects that if nature behaves very similarly to the scenarios one has generated, the optimal strategy will perform well on average. The robust optimization approach attempts to find a more general strategy (one not tied to specific scenarios) that works well in terms of worst-case performance. This is effective in practice, as demonstrated in the computational experiments. Second, the sizes of the resulting optimization problems are very different. Generally, robust optimization formulations based on summary measures of the probability distributions of the uncertainties have a much smaller size than stochastic programming formulations. This allows for the calculations to be run multiple times with little additional computational effort for a range of design parameters for investment products with guarantees, and addresses the problem of the optimal pricing of such contracts as well.

Open Access This article is distributed under the terms of the Creative Commons Attribution 4.0 International License (http://creativecommons.org/licenses/by/4.0/), which permits unrestricted use, distribution, and reproduction in any medium, provided you give appropriate credit to the original author(s) and the source, provide a link to the Creative Commons license, and indicate if changes were made.

\section{References}

Bacinello AR (2003) Fair valuation of a guaranteed life insurance participating contract embedding a surrender option. J Risk Insur 70:461-487

Ben-Tal A, Nemirovski A (1998) Robust convex optimization. Math Oper Res 23(4):769-805 
Ben-Tal A, Margalit T, Nemirovski A (2000) Robust modeling of multi-stage portfolio problems. In: Frenk H, Roos K, Terlaky T, Zhang S (eds) High-performance optimization. Kluwer Academic Publishers, Dordrecht, pp 303-328

Ben-Tal A, El Ghaoui L, Nemirovski A (2009) Robust optimization. Princeton University Press, Princeton

Bertsimas D, Pachamanova D (2008) Robust multiperiod portfolio management in the presence of transaction costs. Comput Oper Res 35:3-17

Bertsimas D, Pachamanova D, Sim M (2004) Robust linear optimization under general norms. Oper Res Lett 32:510-516

Boender G, Dert C, Heemskerk F, Hoek H (2005) A scenario approach of ALM. In: Zenios SA, Ziemba WT (eds) Handbook of asset and liability management. North-Holland, Amsterdam

Brennan MJ, Schwartz ES (1976) The pricing of equity-linked life insurance policies with an asset value guarantee. J Financ Econ 3(3):195-213

Brennan MJ, Schwartz ES (1979) Alternative investment strategies for the issuers of equity linked life insurance policies with an asset value guarantee. J Bus 52(1):63-93

Chen X, Sim M, Sun P (2007) A robust optimization perspective on stochastic programming. Oper Res 55(6):1058-1071

Consigli G, Dempster MAH (1998) Dynamic stochastic programming for asset-liability management. Ann Oper Res 81:131-162

Consiglio A, Cocco F, Zenios SA (2001) The value of integrative risk management for insurance products with guarantees. J Risk Financ 2(3):6-16

Consiglio A, Saunders D, Zenios SA (2006) Asset and liability management for insurance products with minimum guarantees: the UK case. J Bank Financ 30:645-667

Consiglio A, Cocco F, Zenios SA (2008) Asset and liability modeling for participating policies with guarantees. Eur J Oper Res 186(1):380-404

Dantzig GB, Infanger G (1993) Multi-stage stochastic linear programs for portfolio optimization. Ann Oper Res 45:59-76

Duffee G (2002) The long-run behavior of firms stock returns: evidence and interpretations. Working paper, Haas School of Business, University of California at Berkeley, Berkeley, CA

El Ghaoui L, Lebret H (1997) Robust solutions to least-squares problems with uncertain data. SIAM J Matrix Anal Appl 18(4):1035-1064

Escudero LF, Garín A, Merino M, Pérez G (2009) On multistage stochastic integer programming for incorporating logical constraints in asset and liability management under uncertainty. Comput Manag Sci 6:307-327

Fabozzi F, Kolm P, Pachamanova D, Focardi S (2007) Robust portfolio optimization and management. Wiley, Hoboken

Ferstl R, Weissensteiner A (2011) Asset-liability management under time varying investment opportunities. J Bank Financ 35(1):182-192

Gerstner T, Griebel M, Holtz M, Goschnick R, Haep M (2008) A general asset-liability management model for the efficient simulation of portfolios of life insurance policies. Insur Math Econ 42:704-716

Goldfarb D, Iyengar G (2003) Robust portfolio selection problem. Math Oper Res 28(1):1-37

Gondzio J, Kouwenberg R (2001) High performance computing for asset-liability management. Oper Res 49(6):879-891

Goyal A, Welch I (2008) A comprehensive look at the empirical performance of equity premium prediction. Rev Financ Stud 21:1455-1508

Gulpinar N, Pachamanova D (2013) A robust optimization approach to asset-liability management under time-varying investment opportunities. J Bank Financ 37(6):2031-2041

Gulpinar N, Rustem B (2007) Worst-case optimal robust decisions for multi-period portfolio optimization. Eur J Oper Res 183(3):981-1000

Gulpinar N, Rustem B, Settergren R (2004) Simulation and optimization approaches to scenario generation. J Econ Dyn Control 28:1291-1315

Hardy M (2003) Investment guarantees: modeling and risk management for equity-linked life insurance. Wiley, New York

Klaassen P (1998) Financial asset-pricing theory and stochastic programming models for asset-liability management. Manag Sci 44(1):31-48

Kouwenberg R (2001) Scenario generation and stochastic programming models for asset liability management. Eur J Oper Res 134(2):279-292 
Löfberg J (2004) YALMIP: a toolbox for modeling and optimization in MATLAB. In: Proceedings of the CACSD conference, Taipei, Taiwan

Mallier R, Alobaidi G (2002) Pricing equity-linked debt using the Vasicek model. Acta Math Univ Comen 71(2):211-220

Miltersen KR, Persson SA (2003) Guaranteed investment contracts: distributed and undistributed excess return. Scand Actuar J 4:257-279

Natarajan K, Pachamanova D, Sim M (2008) Incorporating asymmetric distributional information in robust value-at-risk optimization. Manag Sci 54(3):573-585

Natarajan K, Pachamanova D, Sim M (2009) Constructing risk measures from uncertainty sets. Oper Res 57(5):1129-1141

Nietert B (2003) Portfolio insurance and model uncertainty. OR Spectr 25:295-316

Oguzsoy CB, Guven S (2007) Robust portfolio planning in the presence of market anomalies. OMEGA: Int J Manag Sci 35(1):1-6

Pachamanova DA, Fabozzi FJ (2016) Portfolio construction and analytics. Wiley, Hoboken

Pae Y, Sabbaghi N (2014) Log-robust portfolio management after transaction costs. OR Spectr 36:95-112

Pinar M (2007) Robust scenario optimization based on downside-risk measure for multi-period portfolio selection. OR Spectr 29(2):295-309

Powell WB (2011) Approximate dynamic programming. Wiley, New York

Ramaswami M, Lieberman PK, Baez G (2001) Equity-linked notes. Global Equity Derivatives, Lehman Brothers

Soyster AL, Murphy FH (2013) A unifying framework for duality and modeling in robust linear programs. OMEGA: Int J Manag Sci 41(6):984-997

Stiefel JD (1984) The guaranteed investment contract (GIC). Soc Actuar 36:265-285

Toy WW, Ryan MD (2000) Public equity-linked debt. In: Francis JC, Toy WW, Whittaker JG (eds) The handbook of equity derivatives. Wiley, New York, pp 329-342

Valle CA, Meade N, Beasley JE (2014) Absolute return portfolios. OMEGA: Int J Manag Sci 45:20-41

Walsh MW (2008) Where did AIG's cash go? The New York Times, October 30

Ziemba WT, Mulvey JM (1998) Worldwide asset and liability modeling. Cambridge University Press, Cambridge 